\title{
1 The restricted nature of protein glycosylation in the mammalian brain
}

2

3 Sarah E. Williams, ${ }^{1,2}$ Maxence Noel, $^{2}$ Sylvain Lehoux, ${ }^{2}$ Murat Cetinbas, ${ }^{3}$ Ramnik J.

4 Xavier, ${ }^{3,4}$ Ruslan Sadreyev, ${ }^{3}$ Edward M. Scolnick, ${ }^{1,4}$ Jordan W. Smoller, ${ }^{1,4}$ Richard D.

5 Cummings, ${ }^{2, \#}$ and Robert G. Mealer ${ }^{1,2,5, \#, *}$

6

$7 \quad$ \# co-senior authors; *corresponding author

$9{ }^{1}$ Psychiatric and Neurodevelopmental Genetics Unit and Department of Psychiatry,

10 Massachusetts General Hospital.

$11{ }^{2}$ Department of Surgery, Beth Israel Deaconess Medical Center. Harvard Medical

12 School, Boston MA.

$13{ }^{3}$ Department of Molecular Biology, Massachusetts General Hospital. Harvard Medical

14 School, Boston, MA.

$15{ }^{4}$ Center for Computational and Integrative Biology, Massachusetts General Hospital.

16 Harvard Medical School, Boston, MA.

$17{ }^{5}$ The Stanley Center for Psychiatric Research at Broad Institute of Harvard/MIT.

18 Cambridge, MA.

20 *Corresponding Author:

21 Robert G. Mealer, M.D., Ph.D.

22 Richard B. Simches Research Center

23185 Cambridge St, $6^{\text {th }}$ Floor 
25 Tel: $+1(617) 724-9076$

26 Email: rmealer@partners.org

Summary

Glycosylation is essential to brain development and function, though prior studies have

31 often been limited to a single analytical technique. Using several methodologies, we

32 analyzed Asn-linked (N-glycans) and Ser/Thr/Tyr-linked (O-glycans) protein

33 glycosylation between brain regions and sexes in mice. Brain $\mathrm{N}$-glycans were

34 surprisingly less complex in sequence and variety compared to other tissues, consisting

35 predominantly of high-mannose precursors and fucosylated/bisected structures. Most

36 brain O-glycans were unbranched, sialylated O-GalNAc and O-mannose structures. A

37 consistent pattern was observed between regions, and sex differences were minimal

38 compared to those observed in plasma. Brain glycans correlate with RNA expression of

39 their synthetic enzymes, and analysis of all glycosylation genes in humans showed a

40 global downregulation in the brain compared to other tissues. We hypothesize that the

41 restricted repertoire of protein glycans arises from their tight regulation in the brain.

42 These results provide a roadmap for future studies of glycosylation in

43 neurodevelopment and disease.

\section{Introduction}


Glycosylation regulates nearly all cellular processes and is particularly important

48 in the development and function of the nervous system (Iqbal et al., 2019; Varki, 2017).

49 Glycans have been shown to influence neurite outgrowth (Gouveia et al., 2012), axon

50 guidance (Bonfanti, 2006), synaptogenesis (Parkinson et al., 2013), membrane

51 excitability (Baycin-Hizal et al., 2014; Isaev et al., 2007; Kulkarni et al., 2020; Weiss et

52 al., 2013), and neurotransmission (Kandel et al., 2018; Scott and Panin, 2014) by

53 modulating the structure, stability, localization, and interaction properties of numerous

54 neuronal proteins. Glycans may consist of a single monosaccharide or can be extended

55 into elaborate sugar oligo/polysaccharides (Varki and Kornfeld, 2017). These structures

56 are covalently attached to lipids or certain amino acids of proteins, which designates

57 protein glycans as either $\mathrm{N}$-glycans or O-glycans. Over 300 enzymes work in an

58 elaborate assembly line to generate, attach, and modify these carbohydrate polymers,

59 creating an immense diversity of glycan structures (Rini and Esko, 2017; Varki, 2017).

Despite its complexity, glycosylation is highly regulated; mutations in a single

61 glyco-gene can lead to profound clinical syndromes, collectively termed congenital

62 disorders of glycosylation (CDGs) (Ng and Freeze, 2018). The majority of CDGs

63 present with neurological symptoms including intellectual disability, seizures, and

64 structural abnormalities, illustrating the particular importance of glycosylation in the

65 brain (Freeze et al., 2015). Fine-tuning of the glycosylation pathway can also affect

66 neurophysiology and behavior, illustrated by the association of several glycosylation

67 enzymes to complex human phenotypes such as schizophrenia (Mealer et al., 2020;

68 Schizophrenia Working Group of the Psychiatric Genomics Consortium, 2014), and

69 intelligence (Hill et al., 2018; Joshi et al., 2018). 
Prior studies of brain glycosylation have typically focused on a single gene,

71 pathway, epitope, or carrier of interest, providing insight into the roles of specific

72 modifications. Glycolipids have been studied extensively, as they comprise the majority

73 of glycan mass in the brain and are crucial for axon myelination, neuronal survival, and

74 regeneration (Hirabayashi, 2012; Schnaar, 2019; Schnaar et al., 2014). Proteoglycans,

75 composed of a core protein modified by various glycosaminoglycan (GAG) chains, have

76 also been a focus, and are known to be temporally and spatially regulated throughout

77 brain development, serving as guidance cues during cell migration and axon pathfinding

78 (Hussain et al., 2006; Irie et al., 2008; Schwartz and Domowicz, 2018). Less attention

79 has been paid to $\mathrm{N}$ - and O-linked protein glycosylation, with a few studies showing the

80 importance of particular modifications such as the Lewis $X$ antigen $\left(L e^{X}\right)$ (Gouveia et al.,

81 2012; Kudo et al., 1998; Pruszak et al., 2009; Sajdel-Sulkowska, 1998), human natural

82 killer antigen (HNK-1) (Morita et al., 2009; Yamamoto et al., 2002), polysialic acid

83 (Hildebrandt and Dityatev, 2013; Sato and Kitajima, 2019), bisecting GlcNAc

84 (Bhattacharyya et al., 2002; Nakano et al., 2019), and O-mannosylation (Bartels et al.,

85 2016; Stalnaker et al., 2011a, 2011b).

Systematic approaches to capture the diversity of all protein glycans in the brain

87 have been attempted using glycomic analysis (Benktander et al., 2018; Gizaw et al.,

88 2015; Ishii et al., 2007; Ji et al., 2015; Stalnaker et al., 2011a), glycoproteomics (Liu et

89 al., 2017; Riley et al., 2019; Trinidad et al., 2013), microarrays (Zou et al., 2017),

90 western blotting (Simon et al., 2019), and MALDI-Imaging techniques (Powers et al.,

91 2013; Toghi Eshghi et al., 2014). While the majority of these have produced

92 complementary results, they tend to be individually limited by sample size, regional 
93 specificity, a single sex, and the technical constraints of a single method. For a more

94 complete picture of brain protein glycosylation, we analyzed the frontal cortex,

95 hippocampus, striatum, and cerebellum of both male and female C57BL/6 mice in the

96 largest such study to date. By comparing results from MALDI-TOF mass spectrometry,

97 tandem mass spectrometry (MS/MS), lectin western blotting, and RNA sequencing, we

98 present a comprehensive portrait of $\mathrm{N}$ - and O-glycosylation in the brain, demonstrating

99 a restricted set of glycans and overall downregulation of the pathway.

100

101 Results

102

103 Protein N-glycosylation shows a unique but consistent pattern across the brain

To assess differences in protein glycosylation across brain regions, we first

105 analyzed $\mathrm{N}$-glycans from the cortex, hippocampus, striatum, and cerebellum of 6 adult

106 male wild-type mice using MALDI-TOF mass spectrometry (MS). Representative MALDI

107 spectra from the four regions show a similar pattern of glycans across the brain (Fig.

108 1A), comparable to human cortex and notably distinct from other tissues such as

109 plasma, CSF, and lung (Fig. S1). Overall, 95 unique N-glycan masses were annotated

110 across the four regions (Table S1). Five of the top 10 most abundant $\mathrm{N}$-glycans in the

111 brain were high-mannose structures, including the most abundant, Man ${ }_{5} \mathrm{GlcNAc}_{2}$ (Man-

112 5), which comprised nearly half of the total glycan signal (Fig. 1B), in contrast to other

113 tissue types which mostly contain more complex, higher molecular weight N-glycans

114 (Fig. S1). 
For further analysis, individual glycans were categorized by monosaccharide composition or shared structural characteristics such as branching (Table S2), and the

117 abundance of these groups were compared between regions. Across the brain, N-

118 glycans were predominantly high-mannose ( $60 \%)$, fucosylated ( 35\%), and bisected

$119(\sim 30 \%)$ structures (Table 1). We noted a low abundance of galactose containing $\mathrm{N}$ -

120 glycans (10-15\%) and an even smaller amount containing sialic acid (1-3\%). Of the few

121 sialylated N-glycans detected, all were modified by the NeuAc form of the sugar.

122 The cerebellum was the most unique of the four brain regions analyzed. Nine of

123 the top ten most abundant $\mathrm{N}$-glycans differed between the cerebellum and other

124 regions, including the most abundant N-glycan, Man-5 (Fig. 1B). The cerebellum also

125 displayed significantly less paucimannose and mono-antennary structures, and greater

126 abundance of complex, multi-antennary, and hybrid glycans (Table 1, Fig. 1C). Of the

127 other brain regions, the cortex and hippocampus appeared most similar in their

128 composition of $\mathrm{N}$-glycans, and trend towards less complex and branched structures

129 than the cerebellum (Table 1, Fig. 1C).

\section{MS/MS confirms common structural components of N-Glycans}

133 arrangements of similar monosaccharide components have the same $\mathrm{m} / \mathrm{z}$, and thus

134 cannot be distinguished by standard mass spectrometry. Therefore, additional analyses 135 are necessary to confirm the structures of glycans detected using MALDI-MS. Tandem 136 mass spectrometry (MS/MS) allows for a single $\mathrm{m} / \mathrm{z}$ peak to be split into fragment ions, 137 which can be analyzed to determine the structure (or structures) of the parent peak in 
138 question. We performed MS/MS on several prominent N-glycans from the cortex and

139 cerebellum and obtained complementary results from both regions. We initially

140 presumed several MALDI-MS peaks corresponded to complex, non-bisected N-glycans

141 commonly found in plasma, as reported in prior studies and other tissues (Benktander

142 et al., 2018; Hu et al., 2013; Ji et al., 2015; Jia et al., 2020; Mealer et al., 2019; Mehta et

143 al., 2012; Palmigiano et al., 2016). However, after additional experiments described

144 below, we determined that these peaks primarily represent bisected and/or hybrid N-

145 glycans, further distinguishing the brain N-glycome from that of other tissues.

147 fragment ions consistent with the predicted high-mannose parent structure, including

148 fragments lacking a single mannose $(\mathrm{m} / \mathrm{z}: 1361)$ or a single GlcNAc $(\mathrm{m} / \mathrm{z}$ : 1302$)$, as well

149 as a free hexose ( $m / z$ : 260) and core GlcNAc ( $m / z: 282,300)$ (Fig. 1D). The peak at

$150 \mathrm{~m} / \mathrm{z}$ : 1835.9 , with an abundance of $\sim 10 \%$ in most brain regions, could represent several

151 potential structures including a biantennary glycan with two terminal GlcNAc residues

152 and a core fucose (FA2) commonly found in plasma. However, MS/MS demonstrated

153 that this glycan has a bisecting GlcNAc residue and only one antenna (Fig. 1E). Several

154 fragment ions from MS/MS results rule in a bisected structure, including a free hexose

155 ( $m / z: 260)$, which indicates the presence of terminal mannose, and the corresponding

156 structure lacking a terminal mannose (m/z: 1617). Additionally, the fragments at $\mathrm{m} / \mathrm{z}$ :

157671 and 949 can only result from the fragmentation of a glycan with a bisecting GlcNAc

158 residue. Glycomic analysis of a mouse lacking Mgat3, which encodes the only enzyme

159 capable of creating bisected N-glycans (GnT-III), showed a dramatic loss of this glycan 
160 in the mutant mouse brain compared to wild-type, providing additional support that $\mathrm{m} / \mathrm{z}$.

1611835 represents a bisected species (Nakano et al., 2019). Additional MS/MS analyses revealed that some peaks consist of a mixture of at

163 least two unique glycans. For example, MS/MS analysis of $\mathrm{m} / \mathrm{z}$ : 2214 generated

164 fragment ions that indicate the presence of both F2A2G1 and F2A1G1B (Fig. 1F). The

165 major peaks m/z: 1576 and 1955 could be formed from either parent structure, but the 166 fragment ions at $m / z$ : 195 and 486 are specific for F2A2G1, while m/z: 671 and 1154

167 are specific for F2A1G1B. The peak at $\mathrm{m} / \mathrm{z}$ : 2244 contained a mixture of bisected hybrid 168 glycans, with either a Lewis $X\left(L e^{X}\right)$ epitope and four mannose residues $(A 1 F G 1 B H 4)$ or

169 a core fucose and five mannose residues (FA1BH5) (Fig. 1G). Fragment ions

170 consistent with A1FG1BH4 include the Le $\mathrm{X}^{\mathrm{X}}$ fragment $(\mathrm{m} / \mathrm{z}$ : 660$)$ and the corresponding

171 glycan missing Le $\mathrm{X}^{\mathrm{X}}(\mathrm{m} / \mathrm{z}$ : 1606). Fragment ions consistent with FA1BH5 include

172 fragments missing both the core GlcNAc and core fucose ( $\mathrm{m} / \mathrm{z}: 1792)$ as well as several 173 smaller fragments resulting from the tri-mannose hybrid arm ( $\mathrm{m} / \mathrm{z}: 196,431$ and 450$)$. In

174 sum, our N-glycan MS/MS results are consistent with the predominance of high-

175 mannose, bisected, and fucosylated structures, lower galactose-containing structures, 176 and a very small amount of sialylated N-glycans in the mouse brain.

178 Endo $\mathrm{H}$ treatment confirms the predominance of high-mannose and hybrid $\mathrm{N}$ -

179 glycans in the brain

180 Given the prevalence of high-mannose N-glycans identified in the brain by

181 MALDI-MS, we sought to confirm this classification with an enzyme that specifically

182 cleaves high-mannose and hybrid structures, known as endoglycosidase $\mathrm{H}$ (Endo $\mathrm{H}$ ). 
183 The intensity of individual N-glycans isolated from the cortex using PNGase F (Fig. 2A)

184 was compared to those present after Endo $\mathrm{H}$ treatment (Fig. 2B) and PNGase F

185 digestion following Endo $\mathrm{H}$ treatment (Fig. 2C). This allowed for the discrimination of

186 structures that are Endo $\mathrm{H}$ sensitive, such as high-mannose and hybrid species, and

187 those that are Endo $\mathrm{H}$ insensitive, such as paucimannose and complex $\mathrm{N}$-glycans. Of

188 note, PNGase $\mathrm{F}$ and Endo $\mathrm{H}$ have a different cleavage site on $\mathrm{N}$-glycans, which results

189 in a difference of one GlcNAc residue between the two digestions and prevents the

190 discernment of structures with and without a core fucose following Endo $\mathrm{H}$ treatment.

191 Due to this limitation, we primarily focused our comparison on the abundance of

192 PNGase F-released glycans before and after Endo $\mathrm{H}$ treatment (Fig. 2A vs 2C) to

193 determine Endo $\mathrm{H}$ sensitivity of each parent peak.

195 glycoproteins in the cortex, as none were detected after subsequent PNGase F

196 treatment (Fig. 2C). Structures corresponding to Man-5-9 were detected in the Endo H

197 spectra, further supporting this conclusion (Fig. 2B). On the contrary, known complex

198 and paucimannose $\mathrm{N}$-glycans were not sensitive to Endo $\mathrm{H}$ treatment; these glycans

199 were present at the same relative intensity after the secondary PNGase F treatment

200 (Fig. 2C), and no structures corresponding to these glycans were detected in the Endo

201 H spectra (Fig. 2B).

Several of the top $15 \mathrm{~N}$-glycan masses identified in the brain have potentially

203 ambiguous structures, as their composition of monosaccharides could form either a

204 hybrid or complex N-glycan structure. For example, the MS peak at $m / z: 2070$

205 (HexNAc4Hex5) could represent a common plasma N-glycan with two antenna and two 
206 terminal galactose residues (A2G2), or a bisected hybrid glycan lacking terminal

207 galactose $(\mathrm{A} 1 \mathrm{BH} 5)$. Endo $\mathrm{H}$ digestion revealed that the $\mathrm{N}-\mathrm{glycan}$ at $\mathrm{m} / \mathrm{z}: 2070$ is

208 predominantly the hybrid species $\mathrm{A} 1 \mathrm{BH} 5$, as its corresponding mass was detected in

209 the Endo H MALDI spectra (Fig. 2B) with minimal signal in the PNGase F spectra after

210 Endo $\mathrm{H}$ treatment (Fig. 2C). In contrast, another potentially ambiguous glycan $(\mathrm{m} / \mathrm{z}$ :

211 2214, denoted as F2A2G1, F2A1G1B) was completely insensitive to Endo H digestion,

212 indicating that glycans at this mass do not include a hybrid species, further supported by

213 our MS/MS results (Fig. 1E).

214 In a third unique case, the peak at $\mathrm{m} / \mathrm{z}$ : 2040 was partially Endo $\mathrm{H}$ sensitive,

215 indicating a mixture of hybrid and non-hybrid glycans present at this mass. The

216 structure corresponding to the parent hybrid glycan FA1BH4 was detected in the Endo

$217 \mathrm{H}$ spectra (A1BH4, Fig. 2B) but a small amount of glycan was present in the secondary

218 PNGase F spectra (Fig. 2C). MS/MS analysis confirmed the presence of both a hybrid

219 structure and a complex, branched structure present at $\mathrm{m} / \mathrm{z}: 2040$, which explains why

220 the signal intensity at this mass decreased after Endo $\mathrm{H}$ treatment but was not removed

221 entirely (Fig. S2).

222

223 Brain O-glycans are primarily sialylated O-GaINAc structures

224 After removal of $\mathrm{N}$-glycans, we analyzed O-glycans isolated from the remaining

225 brain glycopeptides using MALDI-TOF MS. We identified 26 unique O-glycans in at

226 least one brain region, including both O-GalNAc and O-mannose glycans (Table S1).

227 Representative MALDI spectra from the cortex, hippocampus, striatum, and cerebellum

228 showed an overall similar pattern (Fig. 3A). However, there are several differences in 
229 the abundance of individual O-glycans between brain regions, including the most abundant structure, a di-sialylated core 1 O-GalNAc glycan at $\mathrm{m} / z$ : 1256 and the most

231 abundant O-mannose glycan, found at m/z: 1099 (Fig. 3B).

Analysis of all protein O-glycans stratified by structural components (Table S3)

233 revealed that the majority are O-GalNAc-type, comprising $74-84 \%$ of the total O-glycan

234 signal across the brain (Table 1). The abundance of O-mannose species varies

235 significantly between brain regions, ranging from $11 \%$ of all O-glycans in the cortex, to

$23625 \%$ in the cerebellum (Table 1, Fig. 3C). In contrast to brain N-glycans, which have a

237 large amount of fucose $(\sim 30 \%)$ and a paucity of sialic acid $(\sim 2 \%)$, only $\sim 10 \%$ of brain O-

238 glycans are fucosylated, while $\sim 90 \%$ are sialylated. Interestingly, we noted very few O-

239 glycans containing both sialic acid and fucose in the brain (<2\% in all regions). Simple

240 linear regression of fucosylated vs sialylated O-glycans showed a strong and highly

241 significant negative correlation in both O-GalNAc and O-mannose glycans, suggesting

242 competition between these modifications in the brain (Fig. S3). We detected a small

243 amount (1-2\%) of O-glycans containing the NeuGc form of sialic acid, consistent with

244 prior studies (Davies and Varki, 2013; Stalnaker et al., 2011a). Among the dominant O-

245 glycans detected, all of the sialylated species contain solely NeuAc (Fig. 3B). The most

246 common O-glycan structure, $\mathrm{m} / \mathrm{z}$ : 1256.7, comprises $64 \%$ of the total O-glycan

247 abundance and contains two NeuAc residues, while the same structure containing one

248 or two NeuGc residues ( $\mathrm{m} / \mathrm{z}$ : 1286 and 1316$)$ was detected at only $0.8 \%$ and $0.2 \%$

249 abundance, respectively (Table S1, S3).

250

Of note, not all glycans could be classified as O-GalNAc or O-mannose with

251 confidence, since some peaks corresponded to monosaccharide compositions that 
252 could form either type of structure (1-5\% of the total glycan signal). For example, $\mathrm{m} / \mathrm{z}$ :

253 1344, included in the top 10 O-glycans (Fig. 3B), could include both O-mannose and O-

254 GalNAc species, as has been reported in a prior study (Stalnaker et al., 2011a). To

255 further analyze brain O-glycans, we took those that were confirmed as O-GalNAc or O-

256 mannose based on MS/MS results (Fig. 3C-F) or prior reports (Breloy et al., 2012;

257 Stalnaker et al., 2011a) and normalized the abundance within each O-glycan subtype to

258 sort by structural characteristics (Table S3 and Table 1). Further, we excluded potential

259 structures containing the $\alpha$-Gal epitope as our results do not confidently rule in its

260 presence, and we did not detect the transcript for its synthetic enzyme $\alpha 1,3-$

261 galactosyltransferase (Ggta1) in the brain (Huai et al., 2016).

262 Analyzed separately, O-GalNAc and O-mannose glycans both varied in their

263 percentage of core 1 and core 2 structures across brain regions (Table 1, Fig. 3C). The

264 cortex and cerebellum had higher levels of core 2 O-GalNAc glycans than the

265 hippocampus and striatum, as well as lower amounts of NeuAc-containing glycans,

266 since most of the core 2 O-GalNAc glycans do not contain sialic acid $(\mathrm{m} / \mathrm{z}: 1157,779)$.

267 Though the cerebellum had overall more O-mannose glycans than the other brain

268 regions, it has nearly ten-fold less core M2 structures, and a corresponding higher

269 proportion of core M1 structures (Table 1).

$271 \mathrm{MS} / \mathrm{MS}$ confirms the presence of both O-GalNAc and O-mannose glycans in the

272 brain

273 Several of the peaks detected from brain O-glycan samples could correspond to

274 either an O-GaINAc or an O-mannose glycan, so we performed MS/MS of highly 
275 abundant O-glycans to confirm their structural composition (Fig. 3D-G). The most

276 abundant O-glycan peak across all brain regions was $\mathrm{m} / \mathrm{z}$ : 1256 , predicted to represent

277 a core 1 O-GalNAc structure modified by two NeuAc residues. We confirmed the

278 structural composition of this glycan, identifying fragments containing NeuAc bound to

279 both the Gal (m/z: 620) and GalNAc residues ( $m / z: 659)$ (Fig. 3D). The peak at $m / z: 895$

280 also contains a core 1 O-GalNAc glycan, modified by one NeuAc residue, and MS/MS

281 results indicated two structural conformations present. The fragment ions at $\mathrm{m} / \mathrm{z}: 620$,

$282316,298,245$, and 227 all indicate the presence of a core 1 O-glycan with NeuAc

283 attached to the Gal residue. Additionally, the fragments at $m / z: 677,659,284$, and 259

284 correspond to a parent glycan with the sialic acid attached to the core GalNAc residue

285 (Fig. 3E).

The second most abundant O-glycan in the brain, at $\mathrm{m} / \mathrm{z}$ : 1099, could correspond

287 to three possible parent glycans: an O-GalNAc structure modified with one NeuAc and 288 two Gal residues, an O-GalNAc structure with one Gal, one Fuc, and one NeuGc

289 residue, or an O-mannose glycan extended by GlcNAc, Gal and NeuAc. Fragmentation

290 of $\mathrm{m} / \mathrm{z}$ : 1099 revealed fragment ions exclusive to the O-mannose structure, including

291 core Man ( $\mathrm{m} / \mathrm{z}: 275)$ and the corresponding trisaccharide from this cleavage ( $\mathrm{m} / \mathrm{z}: 847$;

292 Fig. 3F). Glycomic analysis of a mouse lacking Pomgnt1, which encodes the enzyme

293 necessary for extension of O-mannose glycans, revealed a complete loss of $\mathrm{m} / \mathrm{z}$ : 1099

294 in the mutant mouse brain, supporting the classification of this peak as an O-mannose

295 structure (Stalnaker et al., 2011a).

296 The peak at $m / z$ : 912 was also absent in the Pomgnt1 knock-out mouse brain,

297 providing evidence that it contains an O-mannose type glycan with a Le $\mathrm{e}^{\mathrm{X}}$ extension, as 
298 opposed to a fucosylated O-GalNAc glycan with the same mass. Our MS/MS results

299 confirmed the structure of the O-mannose glycan at $m / z$ : 912 , with the identification of

300 fragment ions such as the core Man and Le ${ }^{X}$ epitope ( $m / z: 275,660 ;$ Fig. 3G). Of note,

301 the Pomgnt1 knock-out mouse brain retained O-GalNAc glycans, including m/z: 1256

302 and 895, corroborating our determination of these glycans as O-GalNAc-type (Stalnaker

303 et al., 2011a).

304

Sex-specific differences in protein glycosylation are minimal in the brain

306

307

308

309

310

311

312

313

314

315

316

317

318

319

\section{compared to plasma}

Previous studies of the brain glycoproteome have primarily focused on mice of a single sex (Ji et al., 2015; Liu et al., 2017; Riley et al., 2019; Toghi Eshghi et al., 2014;

Zou et al., 2017). However, it is known that mice show both strain specific and sex specific differences in glycosylation of plasma proteins (Reiding et al., 2016). We used MALDI-TOF glycomics to compare the N-glycome of plasma, cortex, and cerebellum in male and female mice, confirming strong sex differences in the plasma $\mathrm{N}$-glycome but only subtle variation in the brain.

In plasma from male and female mice, we detected $29 \mathrm{~N}$-glycans consisting predominantly of complex, sialylated structures modified by the NeuGc form of sialic acid (Table S4, S5), in agreement with previous reports (Reiding et al., 2016). There were striking sex differences in the plasma protein glycomes; notably, the most abundant $\mathrm{N}$-glycan in male mice was A2G2S2 at $\mathrm{m} / \mathrm{z}$ : 2852, while the most abundant $\mathrm{N}$ glycan in female plasma was the fucosylated form of this same species at $\mathrm{m} / \mathrm{z}$ : 3026 
320 (Fig. 4A). In addition to this particular glycan, female mice had a 5-fold increase in all

321 fucosylated structures compared to the male plasma glycome (Table S6).

In the brain, sex differences in protein $\mathrm{N}$-glycosylation were less pronounced,

323 though significant differences were detected. About $15 \%$ of the $\mathrm{N}$-glycans identified in

324 the cortex differed in their abundance compared to the male cortex, including a

325 reduction in Man-7 and Man-8 (Table S4 and Fig. 4B). In the cerebellum, 20\% of N-

326 glycans differed from males, including a significant reduction in FA2B at $\mathrm{m} / \mathrm{z}$ : 2081

327 (Table S4 and Fig. 4C). Based on categorical analysis of these glycans, the female

328 cerebellum showed less biantennary glycans, an increase in sialylation, and an overall

329 trend towards more complex structures compared to the males (Table S6). The cortex

330 followed a similar trend but had overall less distinction between sexes. We anticipate

331 that O-glycosylation differences exist between sexes, similar to N-glycosylation. O-

332 glycans from the cortex of two female mice show minor variations in individual glycan

333 abundance compared to the male cortex O-glycans, but these data were not analyzed

334 further due to low sample size (Fig. S4 and Table S7). Though not as pronounced as

335 the differences observed in plasma, these results illustrate that brain protein

336 glycosylation shows some sex-dependence and underscore the importance of analyzing

337 both sexes separately.

339 Lectin blotting confirms the high abundance of high-mannose, fucosylated, and

340 bisected $\mathrm{N}-\mathrm{glycans}$ in the brain

We next performed western blotting of brain glycoproteins using several

342 commercially available biotinylated lectins. Although lectin binding is often not specific 
343 for a single epitope, their increased affinity for certain glycan features provides

344 important confirmatory information when complemented with techniques such as

345 glycomics and glycosidase sensitivity. Human plasma was included as a positive control

346 given the abundance of literature on the human plasma N-glycome (Clerc et al., 2016).

347 Glycoproteins were treated with or without PNGase F to determine the relative

348 contribution of $\mathrm{N}$ - vs O-glycans to the observed signal. Of note, we detected significant

349 background binding of our fluorescent streptavidin secondary to brain glycoproteins

350 (Fig. S5), which likely resulted from high levels of biotin-bound carboxylases in the brain

351 relative to other tissues as previously described (Grant et al., 2019). To reduce this non-

352 specific binding, we pre-cleared the brain lysates by incubation and precipitation with

353 magnetic streptavidin beads, which removed nearly all non-specific binding and allowed

354 for sensitive detection of glycoprotein bands (Fig. S5).

$355 \quad$ Results from lectin blotting agreed with our N-glycomics, indicating high

356 abundances of high-mannose, fucosylated, and bisected structures, with a near

357 absence of galactosylated and sialylated structures (Fig. 5). Con A, which binds the

358 core mannose structure of all N-glycans, displayed strong binding in the cortex and

359 cerebellum which was completely sensitive to PNGase F cleavage. GNL, also known as

360 snowdrop lectin, primarily binds extended mannose branches found in high-mannose

361 and hybrid N-glycans. Despite minimal binding in plasma, GNL binding of glycoproteins

362 from both brain regions was robust and PNGase F sensitive, corroborating a

363 predominance of these structures in the brain relative to other $\mathrm{N}$-glycans (Fig. 1 and

364 Table 1). AAL binds fucose in both $\square(1-3)$ and $\square(1-6)$ linkages of $N$ - and O-glycans.

365 Strong AAL binding was observed in both brain regions and was entirely PNGase F 
sensitive (Fig. 5), suggesting that the bulk of fucose on glycoproteins in the brain was present on N-glycans, in agreement with our glycomics results (Table 1). PHA-E, commonly used as a marker for bisected N-glycans, showed strong binding in cortex and cerebellum samples and was PNGase F sensitive. RCA binding, which recognizes galactose in both $\beta(1-3)$ and $\beta(1-4)$ linkages, was not detected in brain lysates, but

371 showed strong signal in human plasma, consistent with a relative paucity of galactose

372 terminating glycans in the brain. SNA, also known as elderberry lectin and commonly

373 used to detect glycans with $\square(2-6)$-linked sialic acid, showed only trace binding that was

374 insensitive to PNGase F. This finding is consistent with our glycomics data that only a

375 small minority of $\mathrm{N}$-glycans contain sialic acid ( $2 \%)$, whereas the majority of O-glycans $376(>85 \%)$ contain at least 1 sialic acid residue (Table 1).

Glycosylation gene expression correlates with glycomics and regional differences We next sought to determine if the expression patterns of glycosylation genes

381 would provide insight into the unique glycome patterns observed in the brain.

382 Comprehensive RNA sequencing and analysis was performed using the contralateral

383 hemispheres of cortex and cerebellum from the same male mice used in our glycomic 384 analysis as previously described (Anders et al., 2015; Dobin et al., 2013; Robinson et 385 al., 2010). We generated a list of 269 known glycosyltransferases, glycosylhydrolases, 386 sulfotransferases, and glycan-related genes based on a previous publication (Joshi et 387 al., 2018) and the Carbohydrate Active Enzymes database (CAZy) (Lombard et al., 388 2014), after excluding genes whose transcripts were not detected in our experiment 
(Table S8). A comparison between cortex and cerebellum identified 62 differentially

Several correlates between the unique protein glycome and gene expression in

393 the brain were evident. Of the $\mathrm{N}$-acetylglucosaminyltransferases for N-glycans, Mgat3

394 levels were much higher than those of branching Mgat enzymes (Fig. 6D), consistent with the high abundance of bisected N-glycans and the paucity of complex, branched Nglycans. Of the fucosyltransferases, Fut8 and Fut9 were most abundant (Fig. 6E), correlating with the high amount of core-fucosylated $\mathrm{N}$-glycans and the $\mathrm{Le}^{\mathrm{X}}$ antigen, respectively. Differential expression of several enzymes between cortex and cerebellum also correlated with the glycomics results. For example, the cortex shows higher expression of Mgat5b (Fig. 6F), the sole enzyme responsible for synthesis of core-2 O-

401 Man glycans (Haltiwanger et al., 2017), and these structures were several-fold more 402 abundant in this region.

\section{Human glycosylation genes show a global downregulation in the brain}


412 acetylglucosaminyltransferases (Fig. 6G), fucosyltransferases (Fig. 6H), and the

413 enzymes of O-mannosylation (Fig. 6I). A comparison to other human tissues with well

414 characterized glycomes, such as liver and lung, illustrated the uniqueness of

415 glycosylation gene expression in the brain. Both brain regions express high levels of

416 MGAT3 and have a high abundance of bisected N-glycans, while lung and liver have

417 low levels of MGAT3 and relatively few bisected N-glycans (Fig. 6G) (Jia et al., 2020;

418 Mealer et al., 2019; Mehta et al., 2012). The liver and lung have lower levels of nearly

419 all the enzymes for O-Man synthesis and have relatively few of these structures (Fig.

420 6I).

$421 \quad$ Finally, we compared human glycosylation gene expression in the brain to all

422 other tissues on a global scale (Fig. 7). We applied the publicly available GENE2FUNC

423 feature of the FUMA GWAS platform (Watanabe et al., 2017) to a list of 354 glycan-

424 related genes in humans (Table S9). Comparison of 54 specific tissue types revealed a 425 distinct pattern of downregulation on the individual gene level across 13 brain regions

426 compared to other tissues (Fig. 7A, File S1). Grouped expression analysis of 30

427 general tissue types showed that the brain is the only region with a significantly down-

428 regulated gene set, and the only region which is significantly different when comparing

429 differences in both directions (Fig. 7B). Further analysis of the 13 brain regions as

430 independent tissues shows some regional differences, particularly evident between

431 cortex and cerebellum, though in general the majority of brain regions show an overall

432 downregulation of glycosylation genes (Fig. S6).

434 Discussion 
437 unparalleled level of complexity in its development, organization, and regulation.

438 Glycosylation plays a critical role in the establishment and maintenance of this elaborate

439 network, emphasizing the need to understand the unique glycan species involved.

440 Utilizing MALDI-TOF glycomics, MS/MS, lectin blotting, and RNA sequencing, we have

441 generated a comprehensive map of the predominant $\mathrm{N}$ - and O-linked protein glycans

442 across multiple brain regions and both sexes of mice. Our findings illustrate a relative

443 simplicity of these structures in the brain and a global downregulation of the pathway,

444 suggesting protein glycan synthesis is tightly controlled.

445 The overall pattern of brain glycans, in both mouse and human samples, was

446 markedly distinct from those of other tissues. N-glycomics identified predominantly high-

447 mannose and fucosylated/bisected structures in the mouse brain, with few

448 galactosylated, sialylated, or multi-antennary species present, consistent with results

449 from lectin blotting. Both O-GalNAc and O-mannose glycans were detected in the brain,

450 though the former were several-fold more abundant across all brain regions. O-GalNAc

451 and O-mannose glycans consisted primarily of unbranched core 1 structures (as

452 opposed to extended core 2), and in contrast to $\mathrm{N}$-glycans, were almost entirely

453 sialylated. RNA sequencing suggests that gene expression is at least in part

454 responsible for the unique glycome profile observed in the brain. The cortex,

455 hippocampus, striatum, and cerebellum have overall similar glycomes; however, we

456 identified several glycans, glycan classes, and glycosylation enzymes that differ

457 significantly between brain regions, emphasizing the need to study these regions 
458 indpendently. The cerebellum was the most unique, with more complex, branched, and

459 hybrid N-glycans, as well as the largest proportion of O-mannose species. We detected

460 relatively few differences in brain protein glycosylation between sexes, in contrast to

461 their distinct plasma N-glycomes, suggesting more conserved regulation of

462 glycosylation in the brain.

$463 \quad$ The relative simplicity of brain $\mathrm{N}$-glycans is surprising considering their essential

464 physiological roles. High-mannose N-glycans are often considered immature precursor

465 structures but comprise the majority of all N-glycans in the brain. These structures

466 appear to be mature, as they have been detected on the plasma membrane of neurons

467 as well as on extracellular matrix proteins (Kleene and Schachner, 2004; Schmitz et al.,

468 1993; Tucholski et al., 2013a, 2013b). Some studies have demonstrated that these

469 glycans are involved in cell-cell recognition and homeostatic maintenance, governing

470 the interaction properties of NCAM and basigin and influencing neurite and astrocytic

471 outgrowth (Heller et al., 2003; Horstkorte et al., 1993; Kleene and Schachner, 2004).

472 High-mannose $\mathrm{N}$-glycans are also recognized by the mannose receptor (CD206), a

473 microglia specific receptor that can regulate endocytosis and thus may play a role in

474 synaptic pruning (von Ehr et al., 2020; Martinez-Pomares, 2012; Marzolo et al., 1999;

475 Régnier-Vigouroux, 2003).

476 Of the $\sim 30 \%$ of $\mathrm{N}$-glycans in the brain which are not high-mannose structures,

477 the majority (80-90\%) are bisected. This may contribute to the lack of extended glycans

478 in the brain, as bisection has been shown to impede subsequent modifications of $\mathrm{N}$ -

479 glycans, including galactose and sialic acid, since the additional GlcNAc residue may

480 alter the glycan conformation to prevent interactions with glycosyltransferases (Nagae 
481 et al., 2016; Schachter, 1986). Mgat3 knockout mice develop normally while lacking

482 bisected structures and show a greater relative abundance of complex and modified N-

483 glycans (Nakano et al., 2019). However, high-mannose structures still comprise the

484 majority of $\mathrm{N}$-glycans in the brain of $\mathrm{Mgat}^{--}$mice, suggesting this molecular brake is

485 only one mechanism in place leading to a low abundance of complex N-glycans.

486 O-GalNAc glycans can be extensively modified in other organs (Jin et al., 2017;

487 Wheeler et al., 2019) but are limited to mostly sialylated core 1 structures in the brain.

488 Though they comprise the majority of brain O-glycans, the functional roles of O-GaINAc

489 structures are not well understood in the nervous system. A recent case series identified

490 mutations in GALNT2, one of the 20 enzymes capable of attaching the core GalNAc

491 residue to a serine or threonine, as the cause of a novel CDG (Zilmer et al., 2020).

492 Symptoms include intellectual disability, epilepsy, insomnia, and brain MRI

493 abnormalities, and rodent models of Galnt2 knockout also displayed neurologic

494 abnormalities consistent with a functional role of Galnt2-mediated glycosylation in the

495 brain.

496 O-mannose structures are better understood in terms of their protein carriers and

497 physiological functions, despite their lower abundance (Darula and Medzihradszky,

498 2018; Stalnaker et al., 2011a, 2011b). One common carrier is $\alpha$-dystroglycan, studied

499 extensively in congenital muscular dystrophies, though knockout studies have shown

500 that there are many other proteins modified by O-mannose in the brain (Endo, 2015;

501 Stalnaker et al., 2011a). Extended O-mannose glycans, including those harboring the

502 HNK-1 and Le $\mathrm{e}^{\mathrm{X}}$ epitopes, have been identified on components of perineuronal nets, 503 extracellular matrix structures involved in cell adhesion and neurite outgrowth (Morise et 
504 al., 2014; Pacharra et al., 2013; Yaji et al., 2015). Core M2 glycans have only been

505 reported in the brain, where the key synthetic enzyme MGAT5B is highly enriched, and

506 regulate remyelination, astrocyte activation, and oligodendrocyte differentiation (Inamori

507 et al., 2003; Kanekiyo et al., 2013; Kaneko et al., 2003; Kizuka et al., 2014; Lee et al.,

508 2012). A unique mono-O-mannose glycan on members of the cadherin family has been

509 recently described, and is necessary for the cell-adhesion function of these proteins

510 (Lommel et al., 2013; Vester-Christensen et al., 2013). This O-Man attachment is

511 catalyzed by a novel family of O-mannosyltransferases known as TMTC1-4, rather than

512 the canonical POMT-initiated O-mannose pathway, and is not extended further than the

513 core Man residue (Larsen et al., 2017, 2019).

$514 \quad$ Of note, we do not identify monosaccharide modifications, including mono-O-

515 Man, mono-O-Fuc, or mono-O-GlcNAc, despite brain expression of their synthetic

516 enzymes (Tmtc1-4, Pofut1-2, and Ogt). Such modifications may be present at a lower

517 abundance relative to extended O-GaINAc and O-mannose glycans in the brain, as

518 previous studies have primarily used enrichment strategies for their isolation (Holdener

519 and Haltiwanger, 2019; Larsen et al., 2017; Thompson et al., 2018).

520 While less than $3 \%$ of brain $\mathrm{N}$-glycans are modified by sialic acid, almost all of

521 the O-glycans detected in this study are sialylated. This charged carbohydrate residue

522 has been studied extensively in the context of brain development and disease (Sato and

523 Kitajima, 2019). Sialic acid has been investigated as a regulator of phagocytosis, as

524 microglia express several siglec-type receptors that recognize sialic acid and trigger an

525 inhibitory response in the cell upon binding (Linnartz and Neumann, 2013; Siddiqui et

526 al., 2019). Enzymatic removal of sialic acid from neurons in culture decreases siglec 
527 binding, increases engulfment by microglia, and potentiates complement deposition, a

528 key regulatory step in microglial-mediated synaptic pruning (Linnartz et al., 2012;

529 Schafer et al., 2012; Schizophrenia Working Group of the Psychiatric Genomics

530 Consortium et al., 2016; Stevens et al., 2007; Wielgat and Braszko, 2012). Another

531 carrier of sialic acid in the brain is PSA-NCAM, which can harbor up to 400 sialic acid

532 residues and is critical in brain development and neuronal migration (Nakata and Troy,

533 2005; Schnaar et al., 2014). We did not identify this structure in our samples likely due

534 to its large size and low abundance in the adult brain (Nacher et al., 2013; Quartu et al.,

535 2008; Seki and Arai, 1993).

536 In sum, we present a comprehensive picture of protein N- and O-glycosylation in

537 the mouse brain. Our results highlight unique glycan compositions and distinct

538 regulatory mechanisms across several brain regions, tissue types, and sexes in the

539 largest sample size to date. We emphasized the most abundant $\mathrm{N}$ - and O-glycans in

540 the brain and their potential physiological roles, but this makes no assumption of the

541 function or importance of structures that exist at very low abundance. Subtle changes in

542 glycosylation can lead to major consequences at the protein, cell, and circuit level, so it

543 is essential to understand how such variation is regulated at the genetic (Joshi et al.,

544 2018), epigenetic (Kizuka et al., 2016), transcriptional (Neelamegham and Mahal,

545 2016), developmental (Ishii et al., 2007; Simon et al., 2019), regional (Gizaw et al.,

546 2015; Powers et al., 2014; Toghi Eshghi et al., 2014), and organismal levels (Brown et

547 al., 2019; Gizaw et al., 2016; Yamakawa et al., 2018). The contribution of glycosylation

548 to health and disease has been appreciated in many contexts, especially the nervous

549 system (Reily et al., 2019). In addition to neurologic symptoms of CDGs (Freeze et al., 
550 2015), complex neuropsychiatric phenotypes are linked to glycosylation (Hill et al.,

551 2018; Joshi et al., 2018; Williams et al., 2020). For example, several

552 glycosyltransferases and a missense variant in SLC39A8 are associated with

553 schizophrenia, emphasizing the need for a more detailed understanding of protein

554 glycosylation as it relates to development and disease in the brain (Mealer et al., 2020).

556 Author contributions:

557 SEW performed glycomics experiments, statistical analysis, assisted in RNA analysis,

558 and wrote the manuscript with RGM

559 MN performed lectin blotting, assisted in RNA analysis, and wrote portions of the

560 manuscript

561 SL assisted with experimental methods and advised on analysis of glycans

562 MC performed analysis of RNAseq data

563 RX provided mouse samples for analysis and assisted with project design

564 RS oversaw RNAseq analysis and wrote related portions of the manuscript

565 EMS initiated the project and coordinated collaborations

566 JWS co-supervised SEW and helped conceptualize the project

567 RDC co-supervised SEW and MN, oversaw all experimental analyses, and helped

568 conceptualize the project

569 RGM co-supervised SEW and MN, performed glycomics experiments, lectin blotting,

570 RNA purification and analysis, statistical analysis, helped conceptualize the project, and

571 wrote the manuscript

572 All authors contributed feedback and edits to the manuscript 
574 Acknowledgements: This work was supported by a foundation grant from the Stanley

575 Center for Psychiatric Research at the Broad Institute of Harvard/MIT (awarded to

576 RGM) and NIH grant P41GM103694 (awarded to RDC).

577

578 Declarations of interest: The authors have declared that no conflicts of interest exist.I

Fig. Legends

581

582 Fig. 1. Protein N-glycomics revealed an abundance of high-mannose and

583 fucosylated/bisected structures, confirmed by MS/MS. A) Representative MALDI

584 spectra of protein $\mathrm{N}$-glycans isolated from four brain regions show a similar overall

585 pattern. B) The 10 most abundant $\mathrm{N}$-glycans differ between brain regions ( $\mathrm{n}=6$ each).

586 Data presented as mean percent abundance +/- SEM. Corresponding glycan structures

587 are presented above each peak. For ANOVA $d f=(3,20), F_{\text {crit }}=3.098$. C) Categorical

588 analysis of $\mathrm{N}$-glycans demonstrated greater abundance of complex structures in the

589 cerebellum, with a heat map showing percent change from the average of four regions.

590 D) MS/MS analysis of $m / z: 1579$ generated fragment ions all consistent with Man-5. E)

591 Fragmentation of $\mathrm{m} / \mathrm{z}: 1836$ revealed the presence of a bisected glycan with a terminal

592 mannose. F) Fragmentation of $\mathrm{m} / \mathrm{z}: 2214.1$ and G) $\mathrm{m} / \mathrm{z}: 2244.1$ both indicated two

593 distinct glycan structures, consistent with two parent N-glycans at each peak. CTX =

594 cortex, HIP = hippocampus, STR = striatum, CBLM = cerebellum. 
Fig. 2: Endo $\mathrm{H}$ treatment distinguished high-mannose and hybrid structures from complex and paucimannose $\mathrm{N}$-glycans. The intensity of the 15 most abundant $\mathrm{N}$ -

598 glycans in the cortex is shown after treatment with glycosidases, grouped by high-

599 mannose (green), hybrid (blue), mixed (pink), or complex and paucimannose structures (gray). A) Treatment with PNGase F removed all N-glycans. B) Treatment with Endo H

601 removed only high-mannose and hybrid N-glycans. A placeholder (\#) is included as the same Endo $\mathrm{H}$ fragment can be generated by more than one parent structure if they only differ by the presence of a core fucose. C) PNGase F treatment after Endo $\mathrm{H}$ removed complex and paucimannose $\mathrm{N}$-glycans, which were insensitive to Endo $\mathrm{H}$ treatment.

Fig. 3. Protein O-glycomics revealed a higher proportion of O-GalNAc glycans

608 protein O-glycans isolated from four regions show a consistent pattern across the brain.

B) The 10 most abundant O-glycans include both O-GalNAc and O-mannose type structures $(\mathrm{CTX}=3, \mathrm{HIP}=2, \mathrm{STR}=4, \mathrm{CBLM}=4)$. Data presented as mean percent

611 abundance +/- SEM. Corresponding glycan structures are presented above each peak.

612 For ANOVA $d f=(3,9), F_{\text {crit }}=3.862$. C) Categorical analysis of O-glycans revealed

613 differences in the abundance of O-GalNAc, O-mannose, and NeuGc-containing glycans

614 between regions. Analyzed independently, O-GalNAc and O-mannose glycans varied in

615 their proportion of core 1, core 2, and sialylated structures. Data presented as a heat

616 map showing percent change from the average of four regions. D) MS/MS analysis of

617 the most abundant O-glycan peak, $\mathrm{m} / \mathrm{z}: 1256$, generated fragment ions consistent with

618 a di-sialylated core-1 O-GalNAc glycan. D) Fragmentation of $m / z: 895$ identified ions 
619 representative of mono-sialylated core-1 O-GalNAc glycans with NeuAc attached to

620 either GalNAc or Gal. E) Fragmentation of $m / z: 912$ and F) $m / z: 1099$ confirm the

621 presence of O-mannose glycans with either terminal sialic acid or fucose.

622

623 Fig. 4. Protein N-glycosylation showed minimal sex differences in the brain

624 compared to plasma. Comparison of the top 10 most abundant $\mathrm{N}$-glycans in plasma

625 (A), cortex (B), and cerebellum (C) reveals differences between sexes, with much

626 greater divergence between male and female mice in the plasma than in either brain

627 region. For plasma samples male=8, female=6. For brain samples male=6, female=4.

629 Fig. 5: Lectin blotting supported a predominance of high-mannose, fucose, and

630 bisected $\mathbf{N}-$ glycans in the brain. Protein lysate from mouse cortex and cerebellum

631 with human plasma as a positive control was treated with or without PNGase F and

632 visualized using biotinylated lectins (Con A, GNL, PHA-E, AAL, RCA, and SNA) in

633 addition to immunoblotting for actin and staining for total protein. Non-specific binding of

634 lectins to PNGase F is noted by an asterisk $\left(^{*}\right)$ near $35 \mathrm{kDa}$. A schematic with common

635 lectin-binding sites is shown for reference.

637 Fig. 6. Differential RNA expression between brain regions spans multiple

638 glycosylation pathways and correlates with glycomics results. RNAseq data from

639 cortex and cerebellum ( $n=4$ each) revealed differential expression of enzymes involved

640 in several glycosylation pathways, including the synthesis of N-glycans (A), O-GalNAc

641 glycans (B), and O-mannose glycans (C). Transcripts in red have significantly increased 
642 expression in cortex relative to cerebellum, and those in blue are decreased. ${ }^{*} T m t c 1-4$

643 add O-linked Man but these residues are not extended further. Mouse brain RNAseq

644 results for $\mathrm{N}$-acetylglucosaminyltransferases (D), fucosyltransferases (E), and O-

645 mannose specific enzymes (F) demonstrated high RNA levels of Mgat3, Fut8, Fut9, and

646 Mgat5b, which correlate with results from glycomics. Human RNA seq data showed a

647 similar expression profile for $\mathrm{N}$-acetylglucosaminyltransferases $(\mathrm{G})$, fucosyltransferases

$648(\mathrm{H})$, and O-mannose pathway enzymes $(\mathrm{I})$ in the brain between humans and mice, but

649 this pattern is distinct from human liver and lung.

650

651 Fig. 7. FUMA GENE2FUNC analysis of 354 glycosylation enzymes and related

652 genes in humans revealed a specific downregulation in the brain. A) Heat map

653 demonstrating expression pattern of all glycosylation genes in humans, with a black bar

654 above the 13 columns representing brain regions. Full-size file available in supplement.

655 B) Tissue specific analysis showing down-regulation in the brain compared to all other

65629 tissue types, with significantly enriched DEG sets $\left(P_{\text {bon }}<0.05\right)$ highlighted in red. 


\begin{tabular}{|c|c|c|c|c|c|c|c|}
\hline \multirow{2}{*}{\multicolumn{2}{|c|}{ Glycan Category }} & \multicolumn{4}{|c|}{ Percent Abundance } & \multicolumn{2}{|c|}{ ANOVA Values } \\
\hline & & \multirow{2}{*}{$\begin{array}{c}\text { CTX } \\
3.1 \pm 0.2\end{array}$} & \multirow{2}{*}{$\begin{array}{c}\text { HIP } \\
2.6 \pm 0.3\end{array}$} & \multirow{2}{*}{$\begin{array}{c}\text { STR } \\
2.6 \pm 0.3\end{array}$} & \multirow{2}{*}{$\begin{array}{c}\text { CBLM } \\
1.2 \pm 0.1\end{array}$} & \multirow{2}{*}{$\begin{array}{c}\begin{array}{c}F- \\
\text { value }\end{array} \\
13.23\end{array}$} & \multirow{2}{*}{$\begin{array}{l}\mathrm{p} \text {-value } \\
\mathbf{0 . 0 0 0 0 5}\end{array}$} \\
\hline \multirow{12}{*}{ 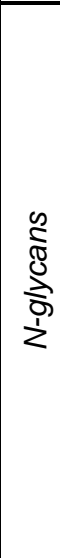 } & Paucimannose & & & & & & \\
\hline & High-mannose & $62.6 \pm 1.3$ & $60.8 \pm 1.8$ & $60.8 \pm 0.6$ & $61.1 \pm 1.8$ & 0.32 & 0.81 \\
\hline & Mono-antennary & $19.0 \pm 0.8$ & $19.2 \pm 0.7$ & $18.9 \pm 0.4$ & $13.0 \pm 0.6$ & 23.39 & 0.000001 \\
\hline & Bi-antennary & $13.6 \pm 0.9$ & $15.5 \pm 1.2$ & $15.4 \pm 0.3$ & $21.7 \pm 0.9$ & 15.68 & 0.00002 \\
\hline & Tri-antennary & $1.7 \pm 0.2$ & $1.7 \pm 0.3$ & $1.9 \pm 0.2$ & $2.6 \pm 0.4$ & 2.46 & 0.09 \\
\hline & Tetra-antennary & $\begin{array}{l}0.14 \pm \\
0.04\end{array}$ & $\begin{array}{l}0.18 \pm \\
0.05\end{array}$ & $\begin{array}{l}0.28 \pm \\
0.07\end{array}$ & $\begin{array}{l}0.32 \pm \\
0.08\end{array}$ & 1.69 & 0.20 \\
\hline & Hybrid & $5.5 \pm 0.2$ & $6.3 \pm 0.2$ & $6.7 \pm 0.4$ & $8.3 \pm 0.4$ & 13.34 & 0.00005 \\
\hline & Bisected & $30.3 \pm 1.1$ & $32.1 \pm 1.5$ & $32.3 \pm 0.5$ & $34.4 \pm 1.5$ & 1.31 & 0.30 \\
\hline & Galactose & $13.5 \pm 0.6$ & $12.8 \pm 1.2$ & $14.1 \pm 0.6$ & $14.1 \pm 1.3$ & 0.40 & 0.76 \\
\hline & Fucose & $34.7 \pm 1.3$ & $36.2 \pm 1.9$ & $36.3 \pm 0.6$ & $35.3 \pm 1.8$ & 0.29 & 0.83 \\
\hline & NeuAc & $1.9 \pm 0.5$ & $2.3 \pm 0.5$ & $2.5 \pm 0.6$ & $2.9 \pm 0.3$ & 0.80 & 0.51 \\
\hline & NeuGc & 0 & 0 & 0 & 0 & 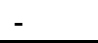 & - \\
\hline \multirow{5}{*}{ 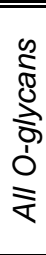 } & "O-GalNAc & $84.1 \pm 0.3$ & 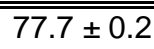 & $82.3 \pm 0.9$ & $=73.5 \pm 2.5$ & 8.83 & 0.005 \\
\hline & O-Mannose & $10.7 \pm 0.4$ & $18.9 \pm 3$ & $13.9 \pm 1.1$ & $25.0 \pm 2.5$ & 14.13 & 0.0009 \\
\hline & Fucose & $9.9 \pm 1.0$ & $7.1 \pm 0.3$ & $7.0 \pm 1.2$ & $10.0 \pm 1.2$ & 1.41 & 0.30 \\
\hline & $\mathrm{NeuAc}$ & $89.2 \pm 0.9$ & $89.7 \pm 0.2$ & $91.4 \pm 1.2$ & $86.6 \pm 1.8$ & 2.30 & 0.15 \\
\hline & NeuGc & $\begin{array}{c}1.28 \pm \\
0.15\end{array}$ & $\begin{array}{c}1.72 \pm \\
0.02\end{array}$ & $2.09 \pm 0.08$ & $\begin{array}{c}2.15 \pm \\
0.15\end{array}$ & 9.56 & 0.004 \\
\hline \multirow{5}{*}{ 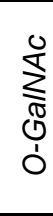 } & $\overline{C O}$ & $90.0 \pm 1.0$ & $994.3 \pm 0.1$ & $94.1 \pm 1.1$ & $90.1 \pm 1.2$ & 4.22 & 0.04 \\
\hline & Core 2 & $10.0 \pm 1.0$ & $5.7 \pm 0.1$ & $5.9 \pm 1.1$ & $9.9 \pm 1.2$ & 4.22 & 0.04 \\
\hline & Fucose & $7.4 \pm 1.0$ & $3.8 \pm 0.2$ & $4.2 \pm 1.1$ & $6.7 \pm 1.4$ & 1.94 & 0.19 \\
\hline & $\mathrm{NeuAc}$ & $90.6 \pm 0.9$ & $94.2 \pm 0.1$ & $94.3 \pm 1.2$ & $89.9 \pm 1.3$ & 4.05 & 0.04 \\
\hline & NeuGc & $1.2 \pm 0.2$ & $1.0 \pm 0.1$ & $1.8 \pm 0.2$ & $2.1 \pm 0.1$ & 11.23 & 0.002 \\
\hline \multirow{5}{*}{ 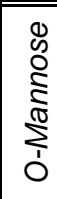 } & Core M1 & $=77.2 \pm 2.4$ & $85.5 \pm 1.4$ & $7 \overline{74.0 \pm 4.1}$ & $\overline{997.0 \pm 0.6}$ & 14.90 & 0.0008 \\
\hline & Core M2 & $22.8 \pm 2.4$ & $14.5 \pm 1.4$ & $26.0 \pm 4.1$ & $3.0 \pm 0.6$ & 14.90 & 0.0008 \\
\hline & Fucose & $22.0 \pm 1.1$ & $20.3 \pm 0.7$ & $21.7 \pm 1.9$ & $19.5 \pm 2.2$ & 0.44 & 0.73 \\
\hline & NeuAc & $78.2 \pm 1.3$ & $76.5 \pm 0.5$ & $77.1 \pm 1.6$ & $78.6 \pm 2.5$ & 0.24 & 0.87 \\
\hline & NeuGc & $2.8 \pm 0.6$ & $5.0 \pm 0.1$ & $4.3 \pm 0.5$ & $2.3 \pm 0.3$ & 7.05 & 0.0098 \\
\hline
\end{tabular}

667 Table 1. Categorical analysis of $\mathrm{N}$ - and $\mathrm{O}$ - glycans highlighted regional

\section{8 differences}




\section{STAR Methods}

\section{RESOURCE AVAILABILITY}

\section{Lead Contact}

679 Further information and requests should be directed to the Lead Contact, Robert Mealer

680 (rmealer@partners.org).

681 Materials Availability

682 This study did not generate any new reagents.

683

\section{Data and Code Availability}

685 This study did not generate any code, and datasets generated from the current study 686 are available from the lead contact upon request.

687

\section{EXPERIMENTAL MODEL AND SUBJECT DETAILS}

690 Fresh post-mortem mouse brain samples were harvested from wild-type mice on a 691 C57BL/6J background originally from The Jackson Laboratory (Cat\#000664) after 692 euthanasia with $\mathrm{CO}_{2}$. Mice from both sexes were used in this study and were 12 weeks

693 old at the time of tissue harvest, sample size specified for each experiment. No live 694 animals were used in this study. All mice were housed and maintained in accordance 695 with the guidelines established by the Animal Care and Use Committee at 
696 Massachusetts General Hospital under protocol \#2003N000158. Human Brain Cerebral

697 Cortex Whole Tissue Lysate was purchased from Novus Biologicals (\#NB820-59182).

\section{METHOD DETAILS}

700 Sample Preparation

701 Brain dissection

702 Following euthanasia with $\mathrm{CO}_{2}$, whole mouse brain was removed and placed on a clean

703 ice cold plastic surface and rinsed with PBS at $4^{\circ} \mathrm{C}$. Four brain regions (frontal cortex, 704 hippocampus, striatum, cerebellum) were isolated from each hemisphere using blunt

705 dissection and placed in $1.5 \mathrm{~mL}$ conical tubes, snap frozen in liquid $\mathrm{N}_{2}$, and stored at $70680^{\circ} \mathrm{C}$ until further use.

707

708 Tissue lysis

709 Frozen brain tissue was lysed in 500 $\mu \mathrm{L}$ ice cold lysis buffer (50 mM TRIS, $150 \mathrm{mM}$

$710 \mathrm{NaCl}, 1.0 \% \mathrm{w} / \mathrm{v}$ Triton-X, pH 7.6) with protease inhibitor (Roche \#46931320019) and

711 dissociated using a hand-held motorized pestle (Kimble \#749540), followed by 2 brief

712 pulses of sonication for 10 seconds with a microtip (Qsonica Q700). An additional

$713500 \mu \mathrm{L}$ of lysis buffer was added to bring the volume to $1 \mathrm{~mL}$, and protein concentration

714 was analyzed using the Pierce BCA Protein Assay Kit (ThermoFisher Scientific

715 \#23255).

716

717 Glycomic Analysis

718 Isolation and purification of glycoproteins 
719 Brain glycoproteins were purified according to standard protocols readily available

720 through the National Center for Functional Glycomics website

721 (www.ncfg.hms.harvard.edu). All buffers were made fresh daily. In brief, 2 mg of protein

722 lysate per sample was dialyzed in $3.5 \mathrm{~L}$ of $50 \mathrm{mM}$ ammonium bicarbonate 3 times at

$7234^{\circ} \mathrm{C}$ over 24 hours using snakeskin dialysis tubing with a molecular cut-off between 1

724 and 5 kDa (ThermoFisher Scientific \#68035). Samples were lyophilized and then

725 resuspended in $1 \mathrm{~mL}$ of $2 \mathrm{mg} / \mathrm{mL}$ 1,4-dithiothreitol (DTT) dissolved in $0.6 \mathrm{M}$ TRIS (pH

726 8.5) and incubated at $50^{\circ} \mathrm{C}$ for 1.5 hours, followed by addition of $1 \mathrm{~mL}$ of $12 \mathrm{mg} / \mathrm{mL}$

727 iodoacetamide in 0.6 M Tris ( $\mathrm{pH} 8.5)$ and incubated at room-temperature for 90 minutes

728 in the dark. Samples were again dialyzed as described above, lyophilized, and

729 resuspended in $1 \mathrm{~mL}$ of $500 \mu \mathrm{g} / \mathrm{ml}$ TPCK-treated trypsin in $50 \mathrm{mM}$ ammonium

730 bicarbonate and incubated overnight (12-16 hours) at $37^{\circ} \mathrm{C}$. Trypsin digestion was

731 stopped by addition of 2 drops 5\% acetic acid, and samples were added to a C18 Sep-

732 Pak (200 mg) column (Waters, \#WAT054945) preconditioned with one column volume

733 each of methanol, 5\% acetic acid, 1-propanol, and 5\% acetic acid. Reaction tubes were

734 washed with $1 \mathrm{~mL} \mathrm{5 \%}$ acetic acid and added to the column, followed by an additional 5

$735 \mathrm{~mL}$ wash of $5 \%$ acetic acid. Each column was placed in a $15 \mathrm{~mL}$ glass tube, and

736 glycopeptides were eluted using $2 \mathrm{~mL}$ of $20 \%$ 1-propanol, $2 \mathrm{~mL}$ of $40 \%$ 1-propanol, and

$7372 \mathrm{~mL}$ of $100 \%$ 1-propanol. The eluted fractions were pooled and placed in a speed

738 vacuum to remove excess organic solvent, followed by lyophilization. 
741 Lyophilized glycopeptides were resuspended in $200 \mu \mathrm{L}$ of $50 \mathrm{mM}$ ammonium

742 bicarbonate and incubated with $3 \mu \mathrm{L}$ of either PNGase F (New England Biolabs,

743 \#P0704) or Endo H (New England Biolabs, \#P0702S) at $37^{\circ} \mathrm{C}$ for 4 hours, then

744 overnight (12-16 hours) with an additional $5 \mu \mathrm{L}$ of enzyme at $37^{\circ} \mathrm{C}$. C18 Sep-Pak

745 columns (200 mg) were preconditioned with one column volume of methanol, 5\% acetic

746 acid, 1-propanol, and 5\% acetic acid and placed in $15 \mathrm{~mL}$ glass tubes. Digested

747 samples were loaded onto preconditioned columns, collecting all flow-through, and N-

748 glycans were eluted with $6 \mathrm{~mL}$ of $5 \%$ acetic acid. Eluted fractions were pooled and

749 placed in a speed vacuum to remove the organic solvents and lyophilized overnight.

750 Glycopeptides remaining on the C18 columns were eluted using $2 \mathrm{~mL}$ of $20 \% 1$ -

751 propanol, $2 \mathrm{~mL}$ of 40\% 1-propanol, and $2 \mathrm{~mL}$ of $100 \%$ 1-propanol, placed in a speed

752 vacuum to remove the organic solvents and lyophilized for O-glycan processing.

753

$754 \quad \beta$-elimination and purification of O-glycans

755 After removing N-glycans from glycopeptides, O-linked glycans were removed using a

$756 \quad \beta$-elimination reaction according to the standard protocols available through the National

757 Center for Functional Glycomics (www.ncfg.hms.harvard.edu). In brief, lyophilized N-

758 glycan-free glycopeptides were resuspended in $400 \mu \mathrm{L}$ of $55 \mathrm{mg} / \mathrm{mL} \mathrm{NaBH} 4$ in $0.1 \mathrm{M}$

$759 \mathrm{NaOH}$ solution and incubated overnight (12-16 hours) at $45^{\circ} \mathrm{C}$. $\beta$-elimination reaction

760 was terminated by adding 4-6 drops of glacial acetic acid to each sample. Desalting

761 columns were prepared using Dowex 50W X8 ion exchange resin with mesh size of

762 200-400 (Sigma-Aldrich, \#44519) in small glass Pasteur pipettes and washed with 10

$763 \mathrm{~mL}$ of $5 \%$ acetic acid. Acetic acid-neutralized samples were loaded onto columns, 
764 collecting flow through in $15 \mathrm{~mL}$ glass tubes. Columns were washed with an additional 3

$765 \mathrm{~mL}$ of $5 \%$ acetic acid and all flow-through was pooled, placed in a speed vacuum to

766 remove organic solvent and lyophilized. Dried samples were resuspended in $1 \mathrm{~mL}$ of

$7671: 9$ acetic acid:methanol solution (v/v=10\%) and dried under a stream of nitrogen,

768 repeating this step an additional three times. C18 Sep-Pak columns (200 mg) were

769 preconditioned with one column volume of methanol, 5\% acetic acid, 1-propanol and

$7705 \%$ acetic acid and placed in $15 \mathrm{~mL}$ glass tubes. The dried samples were resuspended

771 in $200 \mu \mathrm{L}$ of $50 \%$ methanol and loaded onto the conditioned C18 columns, collecting

772 flow-through. Columns were washed with $4 \mathrm{~mL}$ of $5 \%$ acetic acid and all flow-through

773 pooled, placed in a speed vacuum to remove the organic solvents and lyophilized.

774

775 Preparation and isolation of plasma $\mathrm{N}$-glycans

776 Blood samples were collected following CO2 euthanasia and decapitation in a

777 microtainer tube (BD, \#365967), and plasma was separated by centrifugation and

778 stored at $-80^{\circ} \mathrm{C}$ until use. Plasma N-glycan profiling was performed as described

779 previously (Mealer et al., 2019). In brief, $5 \mu \mathrm{L}$ of mouse plasma was lyophilized,

780 resuspended in $20 \mu \mathrm{L} 1 \mathrm{X}$ Rapid PNGase F buffer (NEB \#P0710S), and denatured at

$78170^{\circ} \mathrm{C}$ for $15 \mathrm{~min}$. After cooling to room temperature, $1 \mu \mathrm{L}$ of Rapid PNGase F was

782 added, and incubated at $50^{\circ} \mathrm{C}$ for $60 \mathrm{~min}$. C18 Sep-Pak columns (50 mg, Waters,

783 \#WAT054955) were preconditioned with one column volume of methanol, 5\% acetic

784 acid, 1-propanol, and 5\% acetic acid and placed in $1.5 \mathrm{~mL}$ tubes. PNGase F-treated

785 samples were resuspended in $100 \mu \mathrm{L}$ of $5 \%$ acetic acid and added to the

786 preconditioned columns, collecting all flow-through. The reaction tube was washed with 
787 an additional $100 \mu \mathrm{L}$ of $5 \%$ acetic acid which was added to the column, followed by 1

$788 \mathrm{~mL}$ of $5 \%$ acetic acid, and the entire flow-through was placed in a speed vacuum to

789 remove the organic solvents and lyophilized prior to permethylation as described

790 below.

791

792 Permethylation of $\mathrm{N}$ - and O-glycans

793 Using a clean, dry mortar and pestle, 21 pellets of $\mathrm{NaOH}$ were ground and dissolved

794 into 12 glass pipettes volumes ( 3 ml) of DMSO. A fresh slurry of NaOH/DMSO was

795 made daily. $1 \mathrm{~mL}$ of the slurry was added to the lyophilized $\mathrm{N}$ - and O-glycans in addition

796 to $500 \mu \mathrm{L}$ of iodomethane (Sigma Aldrich, \#289566). Samples were tightly capped and

797 placed on a vortex shaker for 30 minutes at room temperature. After the mixture

798 became white, semi-solid, and chalky, $1 \mathrm{~mL} \mathrm{ddH_{2 }} \mathrm{O}$ was added to stop the reaction and

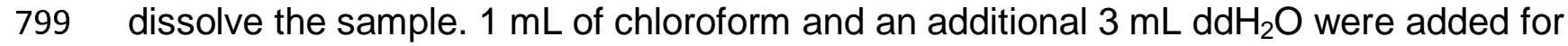
800 chloroform extraction and vortexed followed by brief centrifugation. The aqueous phase

801 was discarded, and the chloroform fraction was washed three additional times with $3 \mathrm{~mL}$

$802 \mathrm{ddH}_{2} \mathrm{O}$. Chloroform was then evaporated in a speed vacuum. Permethylated glycans

803 were resuspended in $200 \mu \mathrm{L}$ of $50 \%$ methanol and added to a C18 Sep-Pak (200 mg)

804 column preconditioned with one column volume each of methanol, $\mathrm{dd}_{2} \mathrm{O}$, acetonitrile,

805 and $\mathrm{ddH}_{2} \mathrm{O}$. The reaction tubes were washed with $1 \mathrm{~mL} 15 \%$ acetonitrile and added to

806 the column, followed by an additional $2 \mathrm{~mL}$ wash of $15 \%$ acetonitrile. Columns were

807 placed into $15 \mathrm{~mL}$ glass round-top tubes, and permethylated glycans were eluted with 3

$808 \mathrm{~mL} 50 \%$ acetonitrile. The eluted fraction was placed in a speed vacuum to remove the

809 acetonitrile and lyophilized overnight. 
811 MALDI-TOF Acquisition

812 Permethylated glycans were resuspended in $25 \mu \mathrm{L}$ of $75 \%$ methanol and spotted in a

$8131: 1$ ratio with DHB matrix on an MTP 384 polished steel target plate (Bruker Daltonics

$814 \quad$ \#8280781) as previously described (Mealer et al., 2019). MALDI-TOF MS data was

815 acquired from a Bruker Ultraflex II instrument using FlexControl Software in the

816 reflective positive mode. For $\mathrm{N}$-glycans, a mass/charge $(\mathrm{m} / \mathrm{z})$ range of $1,000-5,000 \mathrm{kD}$

817 was collected, and for O-glycans, a range of 500-3,000 kD. Twenty independent

818 captures (representing 1,000 shots each) were obtained from each sample and

819 averaged to create the final combined spectra file. Data was exported in .msd format

820 using FlexAnalysis Software for subsequent annotation. Tandem MS (MS/MS) data was

821 collected using the same instrument for both $\mathrm{N}$ - and O-glycans, using the LIFT positive

822 mode, and a +/- 1 Da range from the predicted parent $m / z$, and again represent the sum

823 of twenty independent captures.

$825 \quad$ N- and O-Glycan Analysis

826 Glycans of known structure corresponding to the correct isotopic mass which had a

827 signal to noise ratio greater than $6(\mathrm{~S} / \mathrm{N})$ in at least one brain region averaged over the 828 grouped samples were annotated using mMass software (Strohalm et al., 2010). This

829 resulted in 95 brain $\mathrm{N}$-glycans, 26 brain O-glycans, and 29 plasma N-glycans. The

830 relative abundance of each glycan was calculated as the signal intensity for each

831 isotopic peak divided by the summed signal intensity for all measured glycans within a 832 spectrum. Although using the isotopic mass for quantification may underestimate the 
833 relative abundance of larger glycans given the increased incorporation of Carbon-13,

834 the majority of $\mathrm{N}$ - and all of $\mathrm{O}$ - glycans in the brain are best represented by the isotopic

835 peak $(m / z<2040)$. Structural assignment of glycans was based on MS/MS results,

836 enzyme sensitivity (PNGase F, Endo H), previously confirmed structures (Breloy et al.,

837 2012; Nakano et al., 2019; Stalnaker et al., 2011a), and deductive reasoning when able.

$838 \mathrm{MS} / \mathrm{MS}$ data was annotated by comparing resultant $\mathrm{m} / \mathrm{z}$ peaks to the predicted values

839 for fragment ions with up to 3 bond breaks from all possible parent structures using

840 GlycoWorkbench (Damerell et al., 2012). Brain protein glycans were grouped into

841 different categories based on shared components, such as monosaccharide

842 composition, antennarity, etc., and the summed abundance of each category was

843 compared across brain regions and sexes. All glycan structures are presented

844 according to the Symbol Nomenclature for Glycans (SNFG) guidelines (Neelamegham

845 et al., 2019; Varki et al., 2015) and were drawn using the GlycoGlyph online application

846 (Mehta and Cummings, 2020).

848 Lectin Blotting

849 Brain lysate from the cortex and cerebellum of male mice, were precleared using

850 magnetic streptavidin beads (New England Biolabs, \#S1420S) at a 1:2 ratio of $\mu \mathrm{g}$

851 protein to $\mu \mathrm{L}$ washed beads to decrease background binding resulting from high levels

852 of biotin-bound carboxylases in the brain. After 1-hour incubation at room temperature,

853 beads and biotin-bound proteins were precipitated using a magnetic tube rack, and the

854 supernatant was removed for further analysis. PNGase F sensitivity was determined by

855 incubation of $100 \mu \mathrm{g}$ protein with $5 \mu \mathrm{L}$ PNGase F (New England Biolabs, \#P0704S) at 
$85637^{\circ} \mathrm{C}$ for 1 hour. Lysates were prepared with 4X Sample Loading Buffer (Li-COR, 928-

857 40004) with $10 \%$ v/v $\beta$-mercaptoethanol, and denatured for 10 minutes at $95^{\circ} \mathrm{C}$. For

858 each gel, $15 \mu \mathrm{g}$ protein was loaded per well (NuPAGE 4 to 12\% Bis-Tris, $1.0 \mathrm{~mm}$, Mini

859 Protein Gel, 12-well, ThermoFisher, NP0322). In addition to $2 \mu \mathrm{L}$ Chameleon Duo Pre-

860 Stained Protein Ladder (LiCOR, 928-60000), $50 \mu \mathrm{g}$ of human plasma was loaded as a

861 positive control; plasma is $\sim 60 \%$ is non-glycosylated albumin, thus $\sim 20 \mu \mathrm{g}$ plasma

862 glycoprotein per lane. Gels were run using the MiniProtean Tetra Electrophoresis

863 System (BioRAD, 1658004) at $140 \mathrm{mV}$ for 1 hour. Proteins were transferred to

864 nitrocellulose membranes (ThermoFisher, IB23003) using the iBlot Dry Blotting System

865 (ThermoFisher, IB1001). Membranes were then incubated in 5\% BSA in TBS-Tween

$8660.1 \%$ for 1 hour, followed by incubation with biotinylated lectins (Vector Labs: AAL B-

867 1395, SNA B-1305, GNL B-1245, PHA-E B-1125, RCA B-1085, Con A B-1105) at a

$8681: 1,000$ dilution (1:20,000 for Con A) and 1:2,000 dilution of mouse anti-actin antibody

869 (Abcam, ab8226) in 5\% BSA in TBS-Tween $0.1 \%$, overnight at $4^{\circ} \mathrm{C}$ on a rocking

870 platform shaker. Membranes were washed three times in TBS-Tween $0.1 \%$ for 5

871 minutes, and then incubated with fluorescent conjugated streptavidin IRDye 800CW

872 (LiCOR, 926-32230) and Goat anti-Mouse IgG IRDye 680RD (LiCOR, 925-68070) at

$8731: 25,000$ dilution in 5\% BSA in TBS-Tween $0.1 \%$ for 30 minutes protected from light.

874 Membranes were again washed three times in TBS-Tween $0.1 \%$ for 5 minutes and

875 imaged using a LiCOR Odyssey CLx Imaging System and analyzed using LiCOR Image

876 Studio Software. An identical unprobed membrane was incubated with Revert 700 Total

877 Protein Stain (LiCOR, 926-11011) according to manufacturer's protocol. A

878 comprehensive characterization of biotinylated lectin binding specificity by glycan 
879 microarray can be found on the National Center for Functional Glycomics website

880 (www.ncfg.hms.harvard.edu).

881

882 RNA Sequencing

883 Using the contralateral hemisphere of 4 male mouse brains used in glycomics and lectin

884 blotting experiments, RNA from snap frozen cortex and cerebellum was purified using 885 the RNeasy Lipid Tissue Mini Kit (QIAGEN, 74804) per manufacturer's protocol. RNA-

886 seq libraries were prepared from total RNA using polyA selection followed by the

887 NEBNext Ultra II Directional RNA Library Prep Kit protocol (New England Biolabs,

888 E7760S). Sequencing was performed on Illumina HiSeq 2500 instrument resulting in

889 approximately 30 million of 50 bp reads per sample. Sequencing reads were mapped in

890 a splice-aware fashion to the mouse reference transcriptome ( $\mathrm{mm} 9$ assembly) using

891 STAR (Dobin et al., 2013). Read counts over transcripts were calculated using HTSeq

892 based on the Ensembl annotation for GRCm37/mm9 assembly and presented as

893 Transcripts Per Million (TPM) (Anders et al., 2015). A subset of 269 known

894 glycosyltransferases, glycosylhydrolases, sulfotransferases, and glycan-related genes

895 was created, and differences in expression level between cortex and cerebellum was

896 performed as described below.

897

898 Human RNA comparison and FUMA analysis

899 Performed utilizing publicly available gene expression data from the Genotype-Tissue

900 Expression (GTEx) Portal, Version 8 (https://gtexportal.org). 354 known

901 glycosyltransferases, glycosylhydrolases, sulfotransferases, and glycan-related genes 
902 IDs from humans were used as input into the GENE2FUNC platform of FUMA, which

903 utilizes the GTEx v8 data of both 30 general tissue types, with all brain regions

904 summarized as one tissue type, and 54 specific tissue types that includes 13 individual

905 brain regions. Data is presented alphabetically, with significantly enriched differentially

906 expressed gene sets presented in red after automatic Bonferroni correction with

907 corrected $p<0.05$.

908

909 QUANTIFICATION AND STATISTICAL ANALYSIS

910 For glycomic analyses, statistical analysis of individual and groups of glycans was

911 performed with Microsoft Excel Version 16.35. The abundance of individual glycans and

912 glycan classes were compared between brain regions using single factor ANOVAs.

913 Unpaired t-tests assuming unequal variance were performed for sex comparisons of

914 individual N-glycans and glycan classes from cortex, cerebellum. Significance

915 thresholds for ANOVAs and t-tests were applied at $p<0.05\left(^{*}\right), p<0.01\left(^{* *}\right)$, and $p<$

$\left.9160.001{ }^{* * *}\right)$. A simple regression was performed between O-glycans modified with

917 NeuAc or Fuc using GraphPad Prism v8.4.2. The EdgeR method was used for

918 differential expression analysis of RNAseq data with gene cutoffs of 2-fold change in

919 expression value and false discovery rates (FDR) below 0.05 as previously described

920 (Robinson et al., 2010). Global glycosylation gene regulation in humans was analyzed

921 using the FUMA GWAS GENE2FUC online tool, which identified significantly up- or

922 down-regulated differentially expressed gene sets across human tissue types with a

923 Bonferroni corrected $p$ value $<0.05$ 


\section{Supplementary Files}

926 Supplementary data is presented in attached documents

927 Figs S1-S6 and Tables S1-S7

928 Table S8. RNA expression levels of glycosylation genes detected in cortex and

929 cerebellum

930 Table S9. Glycosylation-related genes included in FUMA analysis

931 File S1: Original FUMA heat map of 354 glycosylation-related gene expression across

932 tissues.

\section{References}

935 Aguet, F., Barbeira, A.N., Bonazzola, R., Brown, A., Castel, S.E., Jo, B., Kasela, S., 936 Kim-Hellmuth, S., Liang, Y., Oliva, M., et al. (2019). The GTEx Consortium atlas of 937 genetic regulatory effects across human tissues (bioRxiv).

Anders, S., Pyl, P.T., and Huber, W. (2015). HTSeq--a Python framework to work with high-throughput sequencing data. Bioinformatics 31, 166-169.

Bartels, M.F., Winterhalter, P.R., Yu, J., Liu, Y., Lommel, M., Möhrlen, F., Hu, H., Feizi, T., Westerlind, U., Ruppert, T., et al. (2016). Protein O-Mannosylation in the Murine Brain: Occurrence of Mono-O-Mannosyl Glycans and Identification of New Substrates.

943 PLOS ONE 11, e0166119.

944 Baycin-Hizal, D., Gottschalk, A., Jacobson, E., Mai, S., Wolozny, D., Zhang, H., Krag, S.S., and Betenbaugh, M.J. (2014). Physiologic and pathophysiologic consequences of altered sialylation and glycosylation on ion channel function. Biochemical and Biophysical Research Communications 453, 243-253.

Benktander, J.D., Gizaw, S.T., Gaunitz, S., and Novotny, M.V. (2018). Analytical with N- and O-Glycans from One Sample. J. Am. Soc. Mass Spectrom. 29, 1125-1137. $\mathrm{N}$-Acetylglucosaminyltransferase III (GlcNAc-TIII) Induces Neurological and Other Traits 953 Absent in Mice That Lack GlcNAc-TIII. J. Biol. Chem. 277, 26300-26309. 
956

957

958

959

960

961

962

963

964

965

966

967

968

969

970

971

972

973

974

975

976

977

978

979

980

981

982

983

984

985

986

987

988

989

990

Breloy, I., Pacharra, S., Aust, C., and Hanisch, F.-G. (2012). A sensitive gel-based global O-glycomics approach reveals high levels of mannosyl glycans in the high mass region of the mouse brain proteome. Biological Chemistry 393, 709-717.

Brown, C.J., Grassmyer, K.T., MacDonald, M.L., Clemmer, D.E., and Trinidad, J.C. (2019). Glycoproteome Analysis of Human Serum and Brain Tissue (bioRxiv).

Clerc, F., Reiding, K.R., Jansen, B.C., Kammeijer, G.S.M., Bondt, A., and Wuhrer, M. (2016). Human plasma protein N-glycosylation. Glycoconj J 33, 309-343.

Damerell, D., Ceroni, A., Maass, K., Ranzinger, R., Dell, A., and Haslam, S.M. (2012). The GlycanBuilder and GlycoWorkbench glycoinformatics tools: updates and new developments. Biological Chemistry 393, 1357-1362.

Darula, Z., and Medzihradszky, K.F. (2018). Analysis of Mammalian O-GlycopeptidesWe Have Made a Good Start, but There is a Long Way to Go. Mol Cell Proteomics 17, 2-17.

Davies, L.R.L., and Varki, A. (2013). Why Is N-Glycolylneuraminic Acid Rare in the Vertebrate Brain? In SialoGlyco Chemistry and Biology I, (Berlin, Heidelberg: Springer Berlin Heidelberg), pp. 31-54.

Dobin, A., Davis, C.A., Schlesinger, F., Drenkow, J., Zaleski, C., Jha, S., Batut, P., Chaisson, M., and Gingeras, T.R. (2013). STAR: ultrafast universal RNA-seq aligner. Bioinformatics 29, 15-21.

eGTEx Project (2017). Enhancing GTEx by bridging the gaps between genotype, gene expression, and disease. Nat Genet 49, 1664-1670.

von Ehr, A., Attaai, A., Neidert, N., Potru, P.S., Ruß, T., Zöller, T., and Spittau, B. (2020). Inhibition of Microglial TGF $\beta$ Signaling Increases Expression of Mrc1. Front. Cell. Neurosci. 14, 66.

Endo, T. (2015). Glycobiology of -dystroglycan and muscular dystrophy. Journal of Biochemistry 157, 1-12.

Freeze, H.H., Eklund, E.A., Ng, B.G., and Patterson, M.C. (2015). Neurological Aspects of Human Glycosylation Disorders. Annu. Rev. Neurosci. 38, 105-125.

Gizaw, S.T., Koda, T., Amano, M., Kamimura, K., Ohashi, T., Hinou, H., and Nishimura, S.-I. (2015). A comprehensive glycome profiling of Huntington's disease transgenic mice. Biochimica et Biophysica Acta (BBA) - General Subjects 1850, 1704-1718.

Gizaw, S.T., Ohashi, T., Tanaka, M., Hinou, H., and Nishimura, S.-I. (2016). Glycoblotting method allows for rapid and efficient glycome profiling of human Alzheimer's disease brain, serum and cerebrospinal fluid towards potential biomarker discovery. Biochimica et Biophysica Acta (BBA) - General Subjects 1860, 1716-1727. 
991 Gouveia, R., Schaffer, L., Papp, S., Grammel, N., Kandzia, S., Head, S.R., Kleene, R., 992 Schachner, M., Conradt, H.S., and Costa, J. (2012). Expression of glycogenes in 993 differentiating human NT2N neurons. Downregulation of fucosyltransferase 9 leads to decreased Lewisx levels and impaired neurite outgrowth. Biochimica et Biophysica Acta (BBA) - General Subjects 1820, 2007-2019.

996 Grant, M.K.O., Shapiro, S.L., Ashe, K.H., Liu, P., and Zahs, K.R. (2019). A Cautionary Tale: Endogenous Biotinylated Proteins and Exogenously-Introduced Protein A Cause 998 Antibody-Independent Artefacts in Western Blot Studies of Brain-Derived Proteins. Biol 999 Proced Online 21, 6.

Haltiwanger, R.S., Wells, L., Freeze, H.H., and Stanley, P. (2017). Chapter 13. Glycosylatransferases and Glycan-Processing Enzymes. In Essentials of Glycobiology, 1002 (Cold Spring Harbor (NY): Cold Spring Harbor Laboratory Press), p.

Heller, M., von der Ohe, M., Kleene, R., Mohajeri, M.H., and Schachner, M. (2003). The immunoglobulin-superfamily molecule basigin is a binding protein for oligomannosidic carbohydrates: an anti-idiotypic approach: Basigin binds to oligomannosidic glycans. Journal of Neurochemistry $84,557-565$.

1007 Hildebrandt, H., and Dityatev, A. (2013). Polysialic Acid in Brain Development and 1008 Synaptic Plasticity. In SialoGlyco Chemistry and Biology I, (Berlin, Heidelberg: Springer 1009 Berlin Heidelberg), pp. 55-96.

Hill, W.D., Arslan, R.C., Xia, C., Luciano, M., Amador, C., Navarro, P., Hayward, C., Nagy, R., Porteous, D.J., Mclntosh, A.M., et al. (2018). Genomic analysis of family data reveals additional genetic effects on intelligence and personality. Mol Psychiatry 23, 2347-2362.

1014 Hirabayashi, Y. (2012). A world of sphingolipids and glycolipids in the brain -Novel functions of simple lipids modified with glucose-. Proc. Jpn. Acad., Ser. B 88, 129143.

1017 Holdener, B.C., and Haltiwanger, R.S. (2019). Protein O-fucosylation: structure and 1018 function. Current Opinion in Structural Biology 56, 78-86.

1019 Horstkorte, R., Schachner, M., Magyar, J.P., Vorherr, T., and Schmitz, B. (1993). The fourth immunoglobulin-like domain of NCAM contains a carbohydrate recognition domain for oligomannosidic glycans implicated in association with L1 and neurite outgrowth. The Journal of Cell Biology 121, 1409-1421.

$1023 \mathrm{Hu}$, Y., Zhou, S., Khalil, S.I., Renteria, C.L., and Mechref, Y. (2013). Glycomic Profiling of Tissue Sections by LC-MS. Anal. Chem. 85, 4074-4079.

1025 Huai, G., Qi, P., Yang, H., and Wang, Y. (2016). Characteristics of a-Gal epitope, anti1026 Gal antibody, a1,3 galactosyltransferase and its clinical exploitation (Review).

1027 International Journal of Molecular Medicine 37, 11-20. 
Hussain, S.-A., Piper, M., Fukuhara, N., Strochlic, L., Cho, G., Howitt, J.A., Ahmed, Y., Powell, A.K., Turnbull, J.E., Holt, C.E., et al. (2006). A Molecular Mechanism for the Heparan Sulfate Dependence of Slit-Robo Signaling. J. Biol. Chem. 281, 39693-39698.

1031

1032

1033

1034

1035

1036

1037

1038

1039

1040

1041

1042

1043

1044

1045

1046

1047

1048

1049

1050

1051

1052

1053

1054

1055

1056

1057

1058

1059

1060

1061

1062

1063

1064

1065

Inamori, K., Endo, T., Ide, Y., Fujii, S., Gu, J., Honke, K., and Taniguchi, N. (2003). Molecular Cloning and Characterization of Human GnT-IX, a Novel $\beta 1,6-\mathrm{N}-$

Acetylglucosaminyltransferase That Is Specifically Expressed in the Brain. J. Biol. Chem. 278, 43102-43109.

Iqbal, S., Ghanimi Fard, M., Everest-Dass, A., Packer, N.H., and Parker, L.M. (2019). Understanding cellular glycan surfaces in the central nervous system. Biochemical Society Transactions 47, 89-100.

Irie, F., Okuno, M., Matsumoto, K., Pasquale, E.B., and Yamaguchi, Y. (2008). Heparan sulfate regulates ephrin-A3/EphA receptor signaling. Proceedings of the National Academy of Sciences 105, 12307-12312.

Isaev, D., Isaeva, E., Shatskih, T., Zhao, Q., Smits, N.C., Shworak, N.W., Khazipov, R., and Holmes, G.L. (2007). Role of Extracellular Sialic Acid in Regulation of Neuronal and Network Excitability in the Rat Hippocampus. Journal of Neuroscience 27, 1158711594.

Ishii, A., Ikeda, T., Hitoshi, S., Fujimoto, I., Torii, T., Sakuma, K., Nakakita, S., Hase, S., and Ikenaka, K. (2007). Developmental changes in the expression of glycogenes and the content of N-glycans in the mouse cerebral cortex. Glycobiology 17, 261-276.

Ji, I.J., Hua, S., Shin, D.H., Seo, N., Hwang, J.Y., Jang, I.-S., Kang, M.-G., Choi, J.-S., and An, H.J. (2015). Spatially-Resolved Exploration of the Mouse Brain Glycome by Tissue Glyco-Capture (TGC) and Nano-LC/MS. Anal. Chem. 87, 2869-2877.

Jia, N., Byrd-Leotis, L., Matsumoto, Y., Gao, C., Wein, A.N., Lobby, J.L., Kohlmeier, J.E., Steinhauer, D.A., and Cummings, R.D. (2020). The Human Lung Glycome Reveals Novel Glycan Ligands for Influenza A Virus. Sci Rep 10, 5320.

Jin, C., Kenny, D.T., Skoog, E.C., Padra, M., Adamczyk, B., Vitizeva, V., Thorell, A., Venkatakrishnan, V., Lindén, S.K., and Karlsson, N.G. (2017). Structural Diversity of Human Gastric Mucin Glycans. Mol Cell Proteomics 16, 743-758.

Joshi, H.J., Hansen, L., Narimatsu, Y., Freeze, H.H., Henrissat, B., Bennett, E., Wandall, H.H., Clausen, H., and Schjoldager, K.T. (2018). Glycosyltransferase genes that cause monogenic congenital disorders of glycosylation are distinct from glycosyltransferase genes associated with complex diseases. Glycobiology 28, 284294.

Kandel, M.B., Yamamoto, S., Midorikawa, R., Morise, J., Wakazono, Y., Oka, S., and Takamiya, K. (2018). N-glycosylation of the AMPA-type glutamate receptor regulates cell surface expression and tetramer formation affecting channel function. J. Neurochem. 147, 730-747. 
1066 Kanekiyo, K., Inamori, K. -i., Kitazume, S., Sato, K., Maeda, J., Higuchi, M., Kizuka, Y., 1067 Korekane, H., Matsuo, I., Honke, K., et al. (2013). Loss of Branched O-Mannosyl 1068 Glycans in Astrocytes Accelerates Remyelination. Journal of Neuroscience 33, 10037106910047.

1070 Kaneko, M., Alvarez-Manilla, G., Kamar, M., Lee, I., Lee, J.-K., Troupe, K., Zhang, W., Osawa, M., and Pierce, M. (2003). A novel $\beta(1,6)-N$-acetylglucosaminyltransferase V (GnT-VB). FEBS Letters 554, 515-519.

1073 Kizuka, Y., Kitazume, S., Okahara, K., Villagra, A., Sotomayor, E.M., and Taniguchi, N. (2014). Epigenetic Regulation of a Brain-specific Glycosyltransferase NAcetylglucosaminyltransferase-IX (GnT-IX) by Specific Chromatin Modifiers. J. Biol. Chem. 289, 11253-11261.

Kizuka, Y., Nakano, M., Miura, Y., and Taniguchi, N. (2016). Epigenetic regulation of neural N-glycomics. Proteomics 16, 2854-2863.

Kleene, R., and Schachner, M. (2004). Glycans and neural cell interactions. Nat Rev Neurosci 5, 195-208.

Kudo, T., Ikehara, Y., Togayachi, A., Kaneko, M., Hiraga, T., Sasaki, K., and Narimatsu, H. (1998). Expression Cloning and Characterization of a Novel Murine a1,3Fucosyltransferase, mFuc-TIX, That Synthesizes the Lewis x (CD15) Epitope in Brain 1084 and Kidney. J. Biol. Chem. 273, 26729-26738. Demonstrates Neuronal Cell-Surface Sialic Acids Modulate Excitability and Network Integration (bioRxiv).

1088 Larsen, I.S.B., Narimatsu, Y., Joshi, H.J., Siukstaite, L., Harrison, O.J., Brasch, J., 1089 Goodman, K.M., Hansen, L., Shapiro, L., Honig, B., et al. (2017). Discovery of an Omannosylation pathway selectively serving cadherins and protocadherins. Proc Natl Acad Sci USA 114, 11163-11168. 56, 171-178.

1095 Lee, J.K., Matthews, R.T., Lim, J.-M., Swanier, K., Wells, L., and Pierce, J.M. (2012). Developmental Expression of the Neuron-specific N-Acetylglucosaminyltransferase $\mathrm{Vb}$ (GnT-Vb/IX) and Identification of Its in Vivo Glycan Products in Comparison with Those of Its Paralog, GnT-V. J. Biol. Chem. 287, 28526-28536.

1099 Linnartz, B., and Neumann, H. (2013). Microglial activatory (immunoreceptor tyrosine1100 based activation motif)- and inhibitory (immunoreceptor tyrosine-based inhibition motif)1101 signaling receptors for recognition of the neuronal glycocalyx. Glia 61, 37-46. 
1102 Linnartz, B., Kopatz, J., Tenner, A.J., and Neumann, H. (2012). Sialic Acid on the 1103 Neuronal Glycocalyx Prevents Complement C1 Binding and Complement Receptor-31104 Mediated Removal by Microglia. Journal of Neuroscience 32, 946-952.

1105

1106

1107

1108

1109
Liu, M.-Q., Zeng, W.-F., Fang, P., Cao, W.-Q., Liu, C., Yan, G.-Q., Zhang, Y., Peng, C., Wu, J.-Q., Zhang, X.-J., et al. (2017). pGlyco 2.0 enables precision N-glycoproteomics with comprehensive quality control and one-step mass spectrometry for intact glycopeptide identification. Nat Commun 8, 438.

Lombard, V., Golaconda Ramulu, H., Drula, E., Coutinho, P.M., and Henrissat, B. (2014). The carbohydrate-active enzymes database (CAZy) in 2013. Nucleic Acids Res. 42, D490-495.

Lommel, M., Winterhalter, P.R., Willer, T., Dahlhoff, M., Schneider, M.R., Bartels, M.F., Renner-Muller, I., Ruppert, T., Wolf, E., and Strahl, S. (2013). Protein O-mannosylation is crucial for E-cadherin-mediated cell adhesion. Proceedings of the National Academy of Sciences 110, 21024-21029.

Lonsdale, J., Thomas, J., Salvatore, M., Phillips, R., Lo, E., Shad, S., Hasz, R., Walters, G., Garcia, F., Young, N., et al. (2013). The Genotype-Tissue Expression (GTEx) project. Nat Genet 45, 580-585.

Martinez-Pomares, L. (2012). The mannose receptor. Journal of Leukocyte Biology 92, 1177-1186.

Marzolo, M.P., von Bernhardi, R., and Inestrosa, N.C. (1999). Mannose receptor is present in a functional state in rat microglial cells. J. Neurosci. Res. 58, 387-395.

Mealer, R.G., Jenkins, B.G., Chen, C.-Y., Daly, M.J., Ge, T., Lehoux, S., Marquardt, T., Palmer, C.D., Park, J.H., Parsons, P.J., et al. (2019). The schizophrenia risk locus in SLC39A8 alters brain metal transport and plasma glycosylation (bioRxiv).

Mealer, R.G., Williams, S.E., Daly, M.J., Scolnick, E.M., Cummings, R.D., and Smoller, J.W. (2020). Glycobiology and schizophrenia: a biological hypothesis emerging from genomic research. Mol Psychiatry.

Mehta, A.Y., and Cummings, R.D. (2020). GlycoGlyph: a glycan visualizing, drawing and naming application. Bioinformatics 36, 3613-3614.

Mehta, A., Norton, P., Liang, H., Comunale, M.A., Wang, M., Rodemich-Betesh, L., Koszycki, A., Noda, K., Miyoshi, E., and Block, T. (2012). Increased Levels of Tetraantennary N-Linked Glycan but Not Core Fucosylation Are Associated with Hepatocellular Carcinoma Tissue. Cancer Epidemiology Biomarkers \& Prevention 21, 925-933.

Morise, J., Kizuka, Y., Yabuno, K., Tonoyama, Y., Hashii, N., Kawasaki, N., Manya, H., Miyagoe-Suzuki, Y., Takeda, S., Endo, T., et al. (2014). Structural and biochemical 
characterization of O-mannose-linked human natural killer-1 glycan expressed on phosphacan in developing mouse brains. Glycobiology 24, 314-324.

1140 Morita, I., Kakuda, S., Takeuchi, Y., Kawasaki, T., and Oka, S. (2009). HNK-1 (human natural killer-1) glyco-epitope is essential for normal spine morphogenesis in developing hippocampal neurons. Neuroscience 164, 1685-1694.

1143 Nacher, J., Guirado, R., and Castillo-Gómez, E. (2013). Structural Plasticity of Interneurons in the Adult Brain: Role of PSA-NCAM and Implications for Psychiatric Disorders. Neurochem Res 38, 1122-1133.

Nagae, M., Kanagawa, M., Morita-Matsumoto, K., Hanashima, S., Kizuka, Y., Taniguchi, N., and Yamaguchi, Y. (2016). Atomic visualization of a flipped-back conformation of bisected glycans bound to specific lectins. Sci Rep 6, 22973.

Nakano, M., Mishra, S.K., Tokoro, Y., Sato, K., Nakajima, K., Yamaguchi, Y., Taniguchi, N., and Kizuka, Y. (2019). Bisecting GlcNAc Is a General Suppressor of Terminal Modification of $N$-glycan. Mol Cell Proteomics 18, 2044-2057.

Nakata, D., and Troy, F.A. (2005). Degree of Polymerization (DP) of Polysialic Acid (PolySia) on Neural Cell Adhesion Molecules (N-CAMs): Development and application of a new strategy to accurately determine the DP of polySia chains on N-CAMs. J. Biol. 1155 Chem. 280, 38305-38316.

1156 Neelamegham, S., and Mahal, L.K. (2016). Multi-level regulation of cellular glycosylation: from genes to transcript to enzyme to structure. Current Opinion in 1158 Structural Biology 40, 145-152.

1159 Neelamegham, S., Aoki-Kinoshita, K., Bolton, E., Frank, M., Lisacek, F., Lütteke, T., 1160 O'Boyle, N., Packer, N.H., Stanley, P., Toukach, P., et al. (2019). Updates to the 1161 Symbol Nomenclature for Glycans guidelines. Glycobiology 29, 620-624.

1162 Ng, B.G., and Freeze, H.H. (2018). Perspectives on Glycosylation and Its Congenital 1163 Disorders. Trends in Genetics 34, 466-476.

1164 Pacharra, S., Hanisch, F.-G., Mühlenhoff, M., Faissner, A., Rauch, U., and Breloy, I. 1165 (2013). The Lecticans of Mammalian Brain Perineural Net Are O-Mannosylated. J. 1166 Proteome Res. 12, 1764-1771.

1167 Palmigiano, A., Barone, R., Sturiale, L., Sanfilippo, C., Bua, R.O., Romeo, D.A., 1168 Messina, A., Capuana, M.L., Maci, T., Le Pira, F., et al. (2016). CSF N-glycoproteomics 1169 for early diagnosis in Alzheimer's disease. Journal of Proteomics 131, 29-37.

1170 Parkinson, W., Dear, M.L., Rushton, E., and Broadie, K. (2013). N-glycosylation 1171 requirements in neuromuscular synaptogenesis. Development 140, 4970-4981.

1172 Powers, T.W., Jones, E.E., Betesh, L.R., Romano, P.R., Gao, P., Copland, J.A., Mehta, 1173 A.S., and Drake, R.R. (2013). Matrix Assisted Laser Desorption Ionization Imaging 
1174 Mass Spectrometry Workflow for Spatial Profiling Analysis of N-Linked Glycan 1175 Expression in Tissues. Anal. Chem. 85, 9799-9806.

1176 Powers, T.W., Neely, B.A., Shao, Y., Tang, H., Troyer, D.A., Mehta, A.S., Haab, B.B., and Drake, R.R. (2014). MALDI Imaging Mass Spectrometry Profiling of N-Glycans in Formalin-Fixed Paraffin Embedded Clinical Tissue Blocks and Tissue Microarrays.

1179 PLoS ONE 9, e106255.

1180 Pruszak, J., Ludwig, W., Blak, A., Alavian, K., and Isacson, O. (2009). CD15, CD24 and 1181 CD29 Define a Surface Biomarker Code for Neural Lineage Differentiation of Stem 1182 Cells. Stem Cells 27, 2928-2940.

1183 Quartu, M., Serra, M.P., Boi, M., Ibba, V., Melis, T., and Del Fiacco, M. (2008). Polysialylated-neural cell adhesion molecule (PSA-NCAM) in the human trigeminal ganglion and brainstem at prenatal and adult ages. BMC Neurosci 9, 108.

1186 Régnier-Vigouroux, A. (2003). The Mannose Receptor in the Brain. In International Review of Cytology, (Elsevier), pp. 321-342.

Reiding, K.R., Hipgrave Ederveen, A.L., Rombouts, Y., and Wuhrer, M. (2016). Murine Plasma N-Glycosylation Traits Associated with Sex and Strain. J. Proteome Res. 15, 3489-3499.

Reily, C., Stewart, T.J., Renfrow, M.B., and Novak, J. (2019). Glycosylation in health and disease. Nat Rev Nephrol 15, 346-366.

Riley, N.M., Hebert, A.S., Westphall, M.S., and Coon, J.J. (2019). Capturing sitespecific heterogeneity with large-scale $\mathrm{N}$-glycoproteome analysis. Nat Commun 10, 1311.

Rini, J.M., and Esko, J.D. (2017). Chapter 6. Glycosylatransferases and GlycanProcessing Enzymes. In Essentials of Glycobiology, (Cold Spring Harbor (NY): Cold Spring Harbor Laboratory Press), p.

Robinson, M.D., McCarthy, D.J., and Smyth, G.K. (2010). edgeR: a Bioconductor package for differential expression analysis of digital gene expression data. Bioinformatics 26, 139-140.

1202 Sajdel-Sulkowska, E.M. (1998). Immunofluorescent detection of CD15-fucosylated 1203 glycoconjugates in primary cerebellar cultures and their function in glial-neuronal 1204 adhesion in the central nervous system. Acta Biochim. Pol. 45, 781-790.

1205 Sato, C., and Kitajima, K. (2019). Sialic Acids in Neurology. In Advances in 1206 Carbohydrate Chemistry and Biochemistry, (Elsevier), pp. 1-64.

1207 Schachter, H. (1986). Biosynthetic controls that determine the branching and 1208 microheterogeneity of protein-bound oligosaccharides. Biochem. Cell Biol. 64, 163-181. 
Schafer, D.P., Lehrman, E.K., Kautzman, A.G., Koyama, R., Mardinly, A.R., Yamasaki, R., Ransohoff, R.M., Greenberg, M.E., Barres, B.A., and Stevens, B. (2012). Microglia Sculpt Postnatal Neural Circuits in an Activity and Complement-Dependent Manner. Neuron 74, 691-705.

Schizophrenia Working Group of the Psychiatric Genomics Consortium (2014). Biological insights from 108 schizophrenia-associated genetic loci. Nature 511, 4211215427.

1216 Schizophrenia Working Group of the Psychiatric Genomics Consortium, Sekar, A., 1217 Bialas, A.R., de Rivera, H., Davis, A., Hammond, T.R., Kamitaki, N., Tooley, K., Presumey, J., Baum, M., et al. (2016). Schizophrenia risk from complex variation of complement component 4. Nature 530, 177-183.

1220 Schmitz, B., Peter-Katalinic, J., Egge, H., and Schachner, M. (1993). Monoclonal antibodies raised against membrane glycoproteins from mouse brain recognize $\mathrm{N}$-linked oligomannosidic glycans. Glycobiology 3, 609-617.

Schnaar, R.L. (2019). The Biology of Gangliosides. In Advances in Carbohydrate Chemistry and Biochemistry, (Elsevier), pp. 113-148. Brain: Gangliosides and Polysialic Acid in Nervous System Development, Stability, Disease, and Regeneration. Physiological Reviews 94, 461-518.

Schwartz, N.B., and Domowicz, M.S. (2018). Proteoglycans in brain development and pathogenesis. FEBS Lett 592, 3791-3805. transmission. Glycobiology 24, 407-417.

1232 Seki, T., and Arai, Y. (1993). Distribution and possible roles of the highly polysialylated neural cell adhesion molecule (NCAM-H) in the developing and adult central nervous system. Neuroscience Research 17, 265-290.

1235 Siddiqui, S.S., Matar, R., Merheb, M., Hodeify, R., Vazhappilly, C.G., Marton, J.,

1238 Simon, F., Bork, K., Gnanapragassam, V.S., Baldensperger, T., Glomb, M.A., Di Sanzo, S., Ori, A., and Horstkorte, R. (2019). Increased Expression of Immature MannoseContaining Glycoproteins and Sialic Acid in Aged Mouse Brains. IJMS 20, 6118.

1241 Stalnaker, S.H., Aoki, K., Lim, J.-M., Porterfield, M., Liu, M., Satz, J.S., Buskirk, S., 1242 Xiong, Y., Zhang, P., Campbell, K.P., et al. (2011a). Glycomic Analyses of Mouse 1243 Models of Congenital Muscular Dystrophy. J. Biol. Chem. 286, 21180-21190. 
Stalnaker, S.H., Stuart, R., and Wells, L. (2011b). Mammalian O-mannosylation: unsolved questions of structure/function. Current Opinion in Structural Biology 21, 603609.

Stevens, B., Allen, N.J., Vazquez, L.E., Howell, G.R., Christopherson, K.S., Nouri, N., Micheva, K.D., Mehalow, A.K., Huberman, A.D., Stafford, B., et al. (2007). The Classical Complement Cascade Mediates CNS Synapse Elimination. Cell 131, 1164-1178.

Strohalm, M., Kavan, D., Novák, P., Volný, M., and Havlíček, V. (2010). mMass 3: A Cross-Platform Software Environment for Precise Analysis of Mass Spectrometric Data. Anal. Chem. 82, 4648-4651.

1256 Toghi Eshghi, S., Yang, S., Wang, X., Shah, P., Li, X., and Zhang, H. (2014). Imaging of $\mathrm{N}$-Linked Glycans from Formalin-Fixed Paraffin-Embedded Tissue Sections Using MALDI Mass Spectrometry. ACS Chem. Biol. 9, 2149-2156.

Trinidad, J.C., Schoepfer, R., Burlingame, A.L., and Medzihradszky, K.F. (2013). N- and O-Glycosylation in the Murine Synaptosome. Mol Cell Proteomics 12, 3474-3488.

1261 Tucholski, J., Simmons, M.S., Pinner, A.L., Haroutunian, V., McCullumsmith, R.E., and 1262 Meador-Woodruff, J.H. (2013a). Abnormal N-linked glycosylation of cortical AMPA 1263 receptor subunits in schizophrenia. Schizophrenia Research 146, 177-183.

1264 Tucholski, J., Simmons, M.S., Pinner, A.L., McMillan, L.D., Haroutunian, V., and Meador-Woodruff, J.H. (2013b). N-linked glycosylation of cortical N-methyl-D-aspartate and kainate receptor subunits in schizophrenia: NeuroReport 24, 688-691.

Varki, A. (2017). Biological roles of glycans. Glycobiology 27, 3-49.

Varki, A., and Kornfeld, S. (2017). Chapter 1. Historical Background and Overview. In Essentials of Glycobiology, (Cold Spring Harbor (NY): Cold Spring Harbor Laboratory Press), p.

1271 Varki, A., Cummings, R.D., Aebi, M., Packer, N.H., Seeberger, P.H., Esko, J.D., 1272 Stanley, P., Hart, G., Darvill, A., Kinoshita, T., et al. (2015). Symbol Nomenclature for 1273 Graphical Representations of Glycans. Glycobiology 25, 1323-1324.

Vester-Christensen, M.B., Halim, A., Joshi, H.J., Steentoft, C., Bennett, E.P., Levery, S.B., Vakhrushev, S.Y., and Clausen, H. (2013). Mining the O-mannose glycoproteome reveals cadherins as major O-mannosylated glycoproteins. Proceedings of the National Academy of Sciences 110, 21018-21023.

1278 Watanabe, K., Taskesen, E., van Bochoven, A., and Posthuma, D. (2017). Functional 1279 mapping and annotation of genetic associations with FUMA. Nat Commun 8, 1826. 
1280 Weiss, N., Black, S.A.G., Bladen, C., Chen, L., and Zamponi, G.W. (2013). Surface expression and function of Cav3.2 T-type calcium channels are controlled by asparagine-linked glycosylation. Pflugers Arch - Eur J Physiol 465, 1159-1170.

Wheeler, K.M., Cárcamo-Oyarce, G., Turner, B.S., Dellos-Nolan, S., Co, J.Y., Lehoux, S., Cummings, R.D., Wozniak, D.J., and Ribbeck, K. (2019). Mucin glycans attenuate the virulence of Pseudomonas aeruginosa in infection. Nat Microbiol 4, 2146-2154.

1286 Wielgat, P., and Braszko, J.J. (2012). The participation of sialic acids in microglia1287 neuron interactions. Cellular Immunology 273, 17-22.

1288 Williams, S.E., Mealer, R.G., Scolnick, E.M., Smoller, J.W., and Cummings, R.D. (2020). Aberrant glycosylation in schizophrenia: a review of 25 years of post-mortem brain studies. Mol Psychiatry.

1291 Yaji, S., Manya, H., Nakagawa, N., Takematsu, H., Endo, T., Kannagi, R., Yoshihara, 1292 T., Asano, M., and Oka, S. (2015). Major glycan structure underlying expression of the 1293 Lewis X epitope in the developing brain is O-mannose-linked glycans on 1294 phosphacan/RPTPß. Glycobiology 25, 376-385.

1295 Yamakawa, N., Vanbeselaere, J., Chang, L.-Y., Yu, S.-Y., Ducrocq, L., Harduin-Lepers, A., Kurata, J., Aoki-Kinoshita, K.F., Sato, C., Khoo, K.-H., et al. (2018). Systems glycomics of adult zebrafish identifies organ-specific sialylation and glycosylation 1298 patterns. Nat Commun 9, 4647.

1299 Yamamoto, S., Oka, S., Inoue, M., Shimuta, M., Manabe, T., Takahashi, H., Miyamoto, M., Asano, M., Sakagami, J., Sudo, K., et al. (2002). Mice Deficient in Nervous Systemspecific Carbohydrate Epitope HNK-1 Exhibit Impaired Synaptic Plasticity and Spatial 1302 Learning. J. Biol. Chem. 277, 27227-27231.

1303 Zilmer, M., Edmondson, A.C., Khetarpal, S.A., Alesi, V., Zaki, M.S., Rostasy, K., Madsen, C.G., Lepri, F.R., Sinibaldi, L., Cusmai, R., et al. (2020). Novel congenital disorder of O-linked glycosylation caused by GALNT2 loss of function. Brain 143, 11141126.

1307 Zou, X., Yoshida, M., Nagai-Okatani, C., Iwaki, J., Matsuda, A., Tan, B., Hagiwara, K., 1308 Sato, T., Itakura, Y., Noro, E., et al. (2017). A standardized method for lectin microarray1309 based tissue glycome mapping. Sci Rep 7, 43560. 
bioRxiv preprint doi: https://doi.org/10.1101/2020.10.01.322537; this version posted October 1, 2020. The copyright holder for this preprint
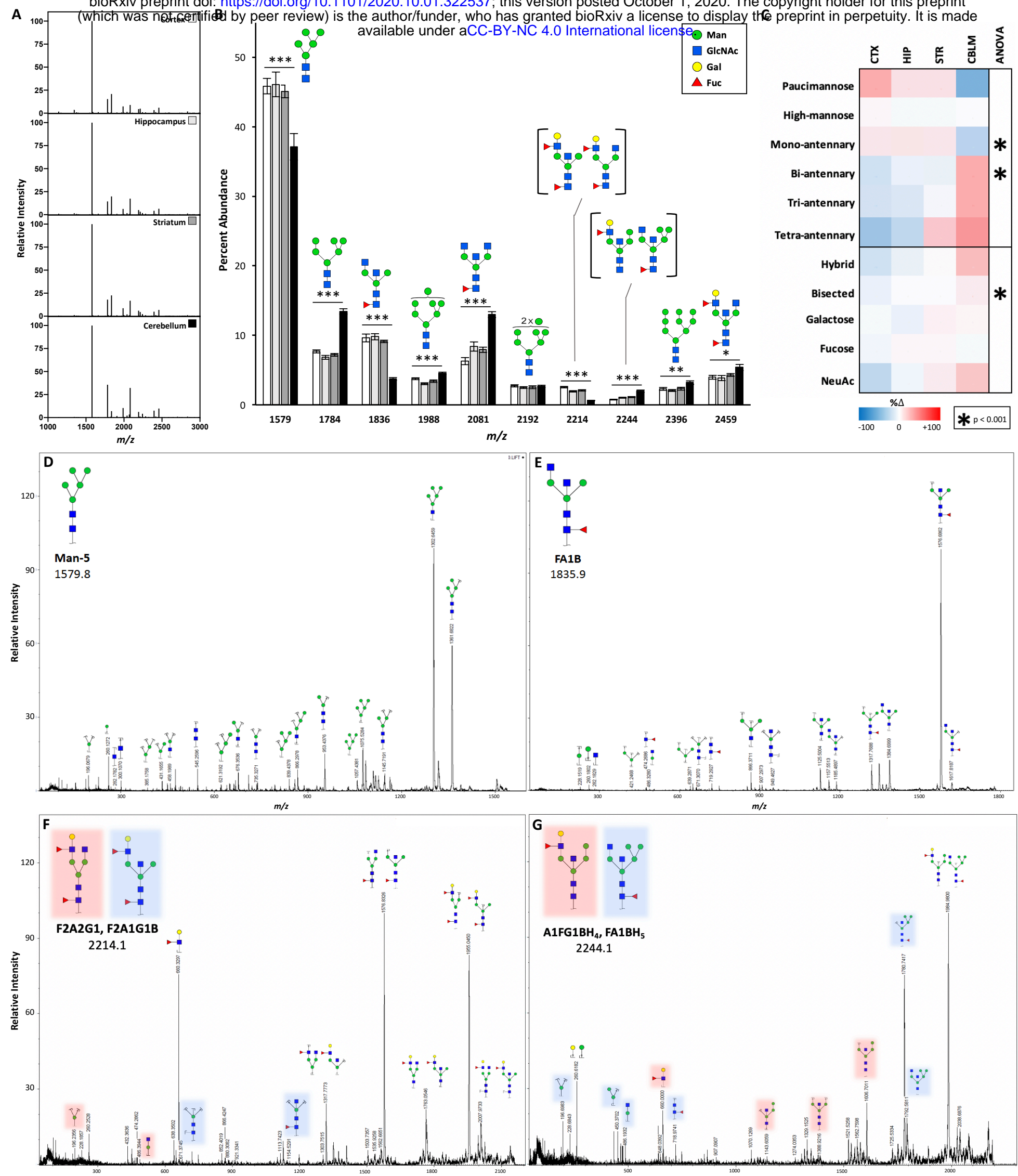

Fig. 1. Protein $\mathrm{N}$-glycomic $\mathrm{s}^{/ 2}$ revealed an abundance of high-mannose and fucos/zylated/bisected structures, confirmed by MS/MS. A) Representative MALDI spectra of protein N-glycans isolated from four brain regions show a similar overall pattern. B) The 10 most abundant $\mathrm{N}$-glycans differ between brain regions ( $\mathrm{n}=6$ each). Data presented as mean percent abundance +/- SEM. Corresponding glycan structures are presented above each peak. For ANOVA calculations, $d f=(3,20), \mathrm{F}_{\text {crit }}=3.098$. C) Categorical analysis of $\mathrm{N}$-glycans demonstrates greater abundance of complex structures in the cerebellum, with a heat map showing percent change from the average of four regions. D) MS/MS analysis of $\mathrm{m} / \mathrm{z}$ : 1579 generated fragment ions all consistent with Man-5. E) Fragmentation of $\mathrm{m} / \mathrm{z}$ : 1836 revealed the presence of a bisected glycan with a terminal mannose. F) Fragmentation of $\mathrm{m} / \mathrm{z}: 2214.1$ and $\mathrm{G}$ ) $\mathrm{m} / \mathrm{z}$ : 2244.1 both indicated two distinct glycan structures, consistent with two parent $\mathrm{N}$-glycans at each peak. CTX $=$ cortex, HIP = hippocampus, STR = striatum, CBLM = cerebellum. 


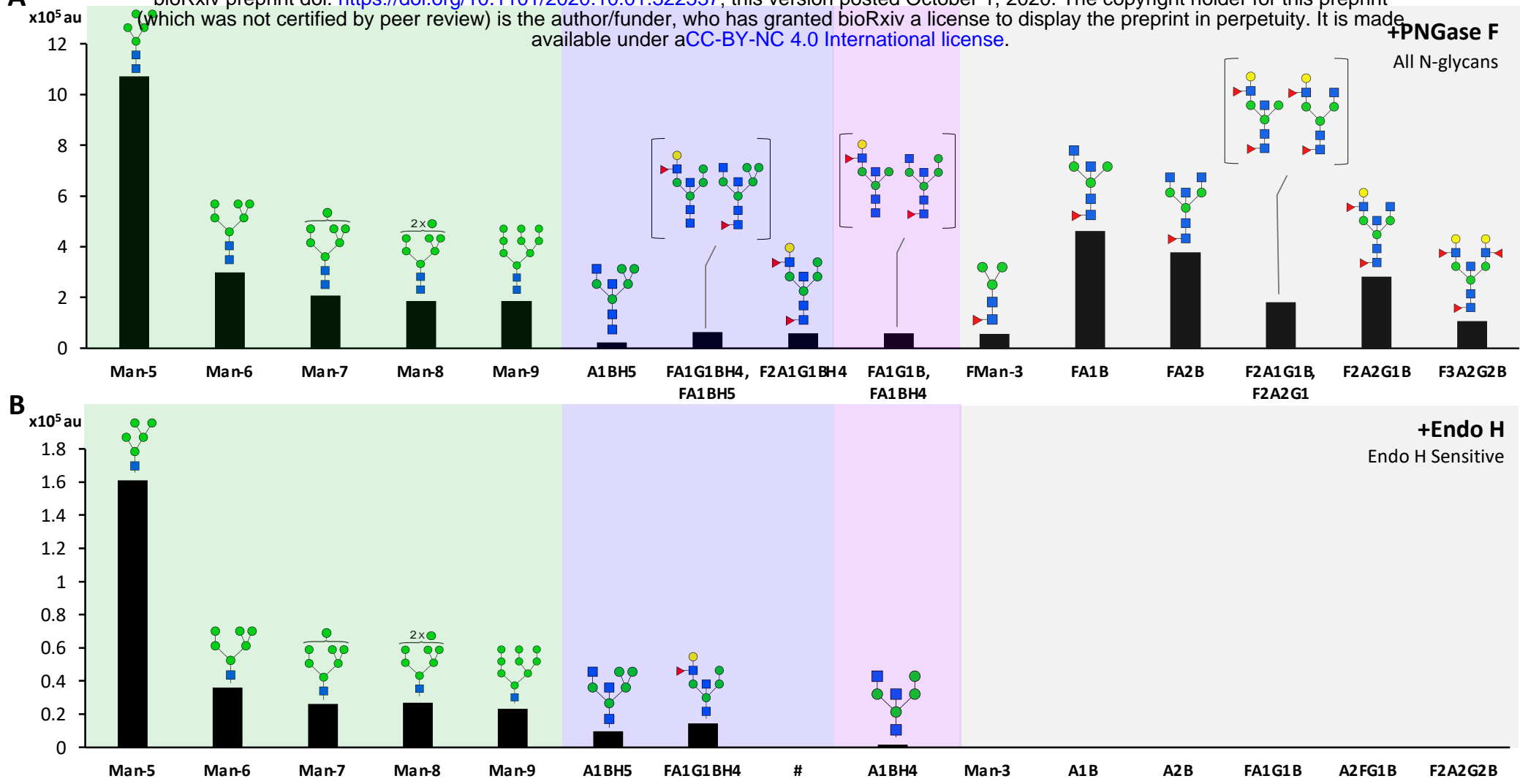

C



$\square$ High-Mannose N-glycans $\square$ Hybrid N-glycans $\square$ Mixture of Hybrid, Complex N-glycans $\square$ Complex and Paucimannose N-glycans

Fig. 2: Endo $H$ treatment distinguished high-mannose and hybrid structures from complex and paucimannose $\mathbf{N}$-glycans. The intensity of the 15 most abundant $\mathrm{N}$-glycans in the cortex is shown after treatment with different glycosidases, grouped by high-mannose (green), hybrid (blue), mixed (pink), or complex and paucimannose structures (gray). A) Treatment with PNGase F removed all N-glycans. B) Treatment with Endo $\mathrm{H}$ removed only high-mannose and hybrid $\mathrm{N}$-glycans. A placeholder (\#) is included as the same Endo $\mathrm{H}$ fragment can be generated by more than one parent structure if they only differ by the presence of a core fucose, which is removed by Endo $\mathrm{H}$. C) PNGase $\mathrm{F}$ treatment after Endo $\mathrm{H}$ removed complex and paucimannose $\mathrm{N}$-glycans, which were insensitive to Endo $\mathrm{H}$ treatment. 
A ${ }_{100} \square$ cortex bioRxiv preprint dpi: $h$ Bps://doi.org/10.1101/2020.10.01.322537; this version posted October 1, 2020. The copyrght holder for this preprint
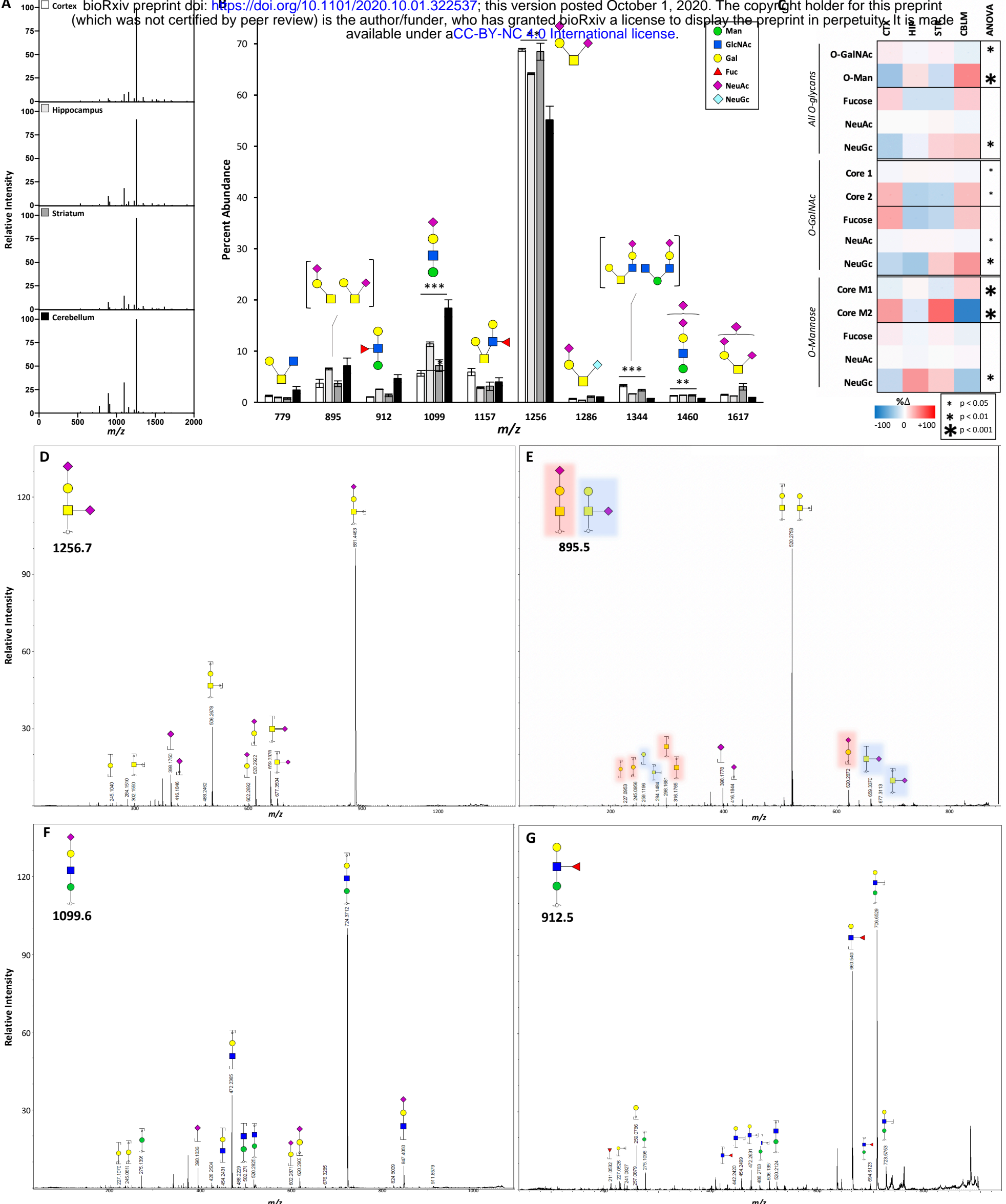

Fig. 3. Protein O-glycomics revealed a higher proportion of O-GalNAc glycans compared to O-Man, confirmed by MS/MS. A) Representative MALDI spectra of protein O-glycans isolated from four regions show a consistent pattern across the brain. B) The 10 most abundant O-glycans in the brain include both O-GalNAc and O-mannose type structures (CTX=3, HIP=2, STR=4, CBLM=4). Data presented as mean percent abundance +/- SEM. Corresponding glycan structures are presented above each peak. For ANOVA calculations, $d f=(3,9), F_{\text {crit }}=3.862$. C) Categorical analysis of $O-g l y c a n s$ revealed differences in the abundance of O-GalNAc, O-mannose, and NeuGc-containing glycans between regions. Analyzed independently, O-GalNAc and O-mannose glycans varied in their proportion of core 1, core 2, and sialylated structures. Data presented as a heat map showing percent change from the average of four brain regions. D) MS/MS analysis of the most abundant O-glycan peak, $\mathrm{m} / \mathrm{z}: 1256$, generated fragment ions consistent with a di-sialylated core-1 O-GalNAc glycan. D) Fragmentation of $\mathrm{m} / \mathrm{z}$ : 895 identified ions representative of mono-sialylated core-1 O-GalNAc glycans with NeuAc attached to either GalNAc or Gal. E) Fragmentation of $\mathrm{m} / \mathrm{z}$ : 912 and F) $\mathrm{m} / \mathrm{z}$ : 1099 confirm the presence of O-mannose glycans with either terminal sialic acid or fucose. 
bioRxiv preprint doi: https://doi.org/10.1101/2020.10.01.322537; this version posted October 1, 2020. The copyright holder for this preprint

(which was not certified by peer review) is the author/funder, who has granted bioRxiv a license to display the preprint in perpetuity. It is made
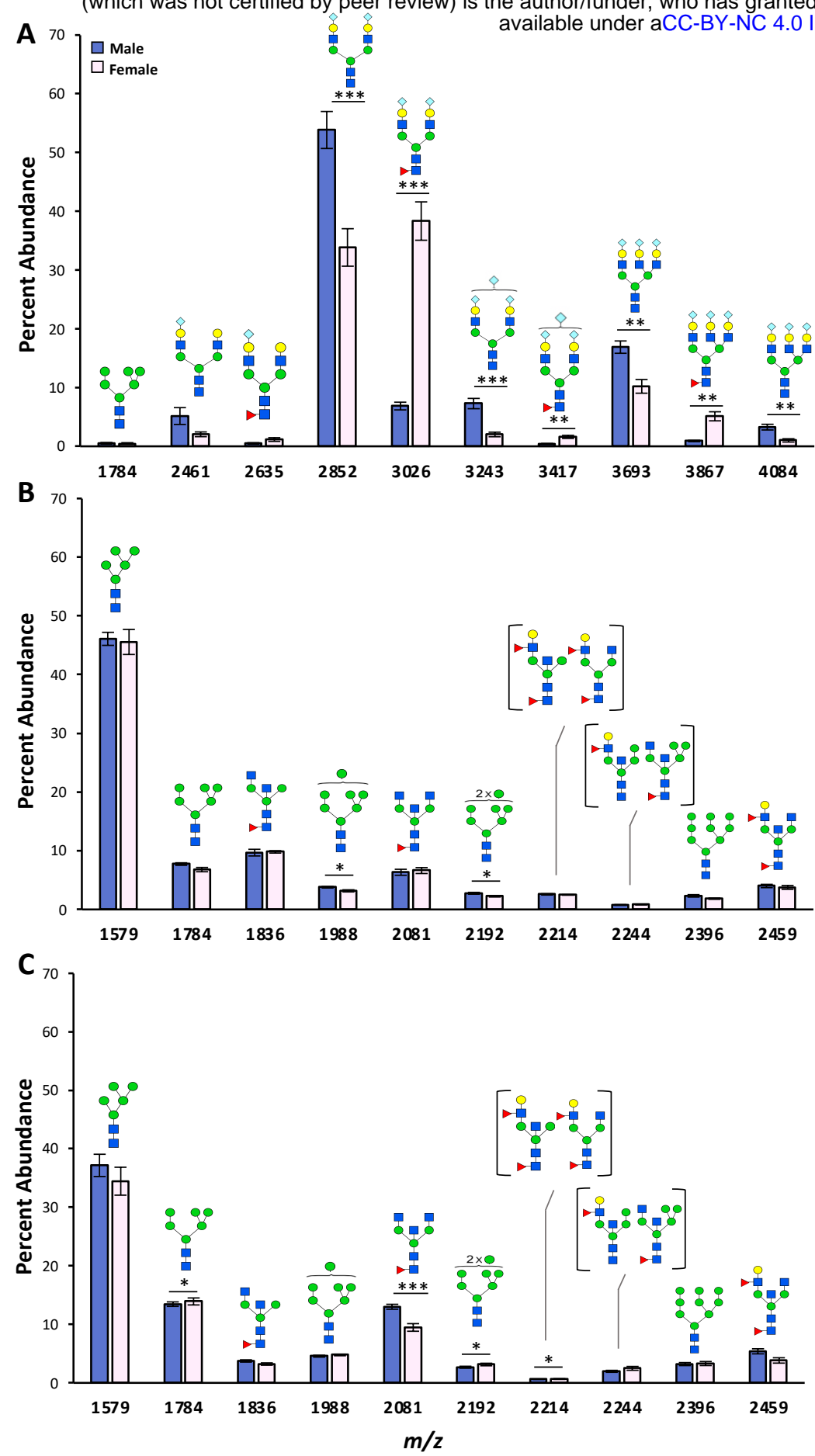

Fig. 4. Protein $\mathrm{N}$-glycosylation showed minimal sex differences in the brain compared to plasma. Comparison of the top 10 most abundant protein $\mathrm{N}$ glycans in plasma (A), cortex (B), and cerebellum (C) reveals differences between sexes, with much greater divergence between male and female mice in the plasma than in either brain region. For plasma samples, male $=8$, female $=6$. For brain samples, male $=6$, female $=4$. 

available under aCC-BY-NC 4.0 International license.

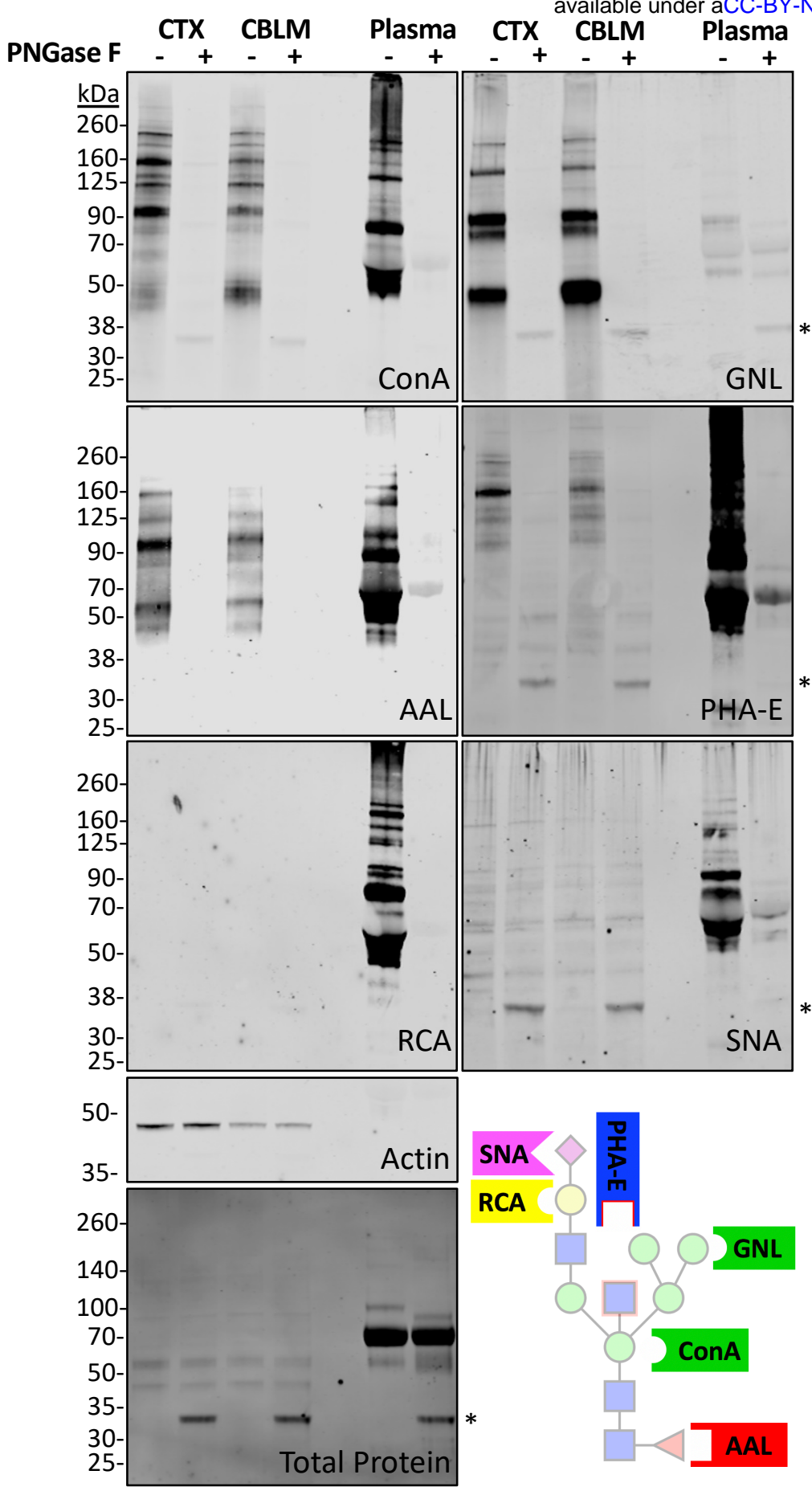

Fig. 5: Lectin blotting supports a predominance of high-mannose, fucose, and bisected $\mathrm{N}$-glycans in the brain. Protein lysate from mouse cortex and cerebellum with human plasma as a positive control was treated with or without $P N G$ ase $F$ and visualized using biotinylated lectins (Con A, GNL, PHA-E, AAL, RCA, and SNA) in addition to immunoblotting for actin and staining for total protein. Non-specific binding of lectins to PNGase $\mathrm{F}$ is noted by an asterisk (*) near $35 \mathrm{kDa}$. A schematic with common lectin-binding sites is shown for reference. 
bioRxiv preprint doi: https://doi.org/10.1101/2020.10.01.322537; this version posted October 1, 2020. The copyright holder for this preprint (which was not certified by peer review) is the author/funder, who has granted bioRxiv a license to display the preprint in perpetuity. It is made
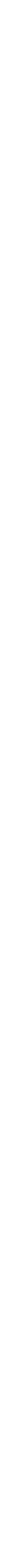

Fig. 6. Differential RNA expression between brain regions spans multiple glycosylation pathways and correlates with glycomics results. RNA seq data from cortex and cerebellum ( $n=4$ each) revealed differential expression of enzymes involved in several glycosylation pathways, including the synthesis of N-glycans (A), OGalNAc glycans (B), and O-mannose glycans (C). Transcripts in red have significantly increased expression in cortex relative to cerebellum, and those in blue are decreased. ${ }^{*}$ Tmtc1-4 add O-linked Man but these residues are not extended further. Mouse brain RNA seq results for $\mathrm{N}$-acetylglucosaminyltransferases (D), fucosyltransferases (E), and O-mannose specific enzymes (F) demonstrated high RNA levels of Mgat3, Fut8, Fut9, and Mgat5b, which correlate with results from glycomics. Human RNA seq data showed a similar expression profile for $\mathrm{N}$-acetylglucosaminyltransferases $(\mathrm{G})$, fucosyltransferases $(\mathrm{H})$, and $\mathrm{O}$-mannose pathway enzymes (I) in the brain between humans and mice, but this pattern is distinct from human liver and lung. 
bioRxiv preprint doi: https://doi.org/10.1101/2020.10.01.322537; this version posted October 1,2020. The copyright holder for this preprint (which was not certified by peer review) is the author/funder, who has granted bioRxiv a license to display the preprint in perpetuity. It is made

A

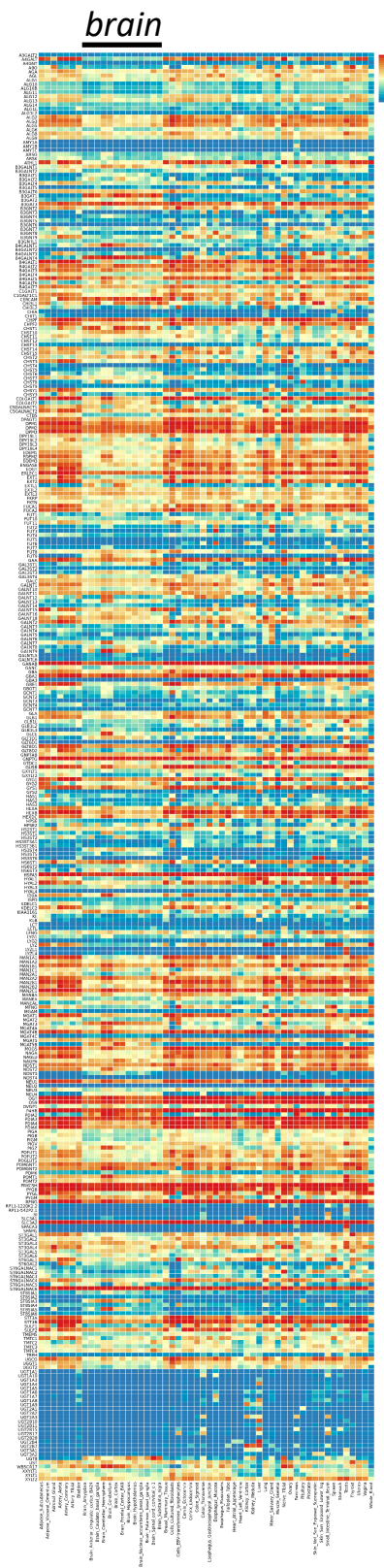

B available under aCC-BY-NC 4.0 International license.

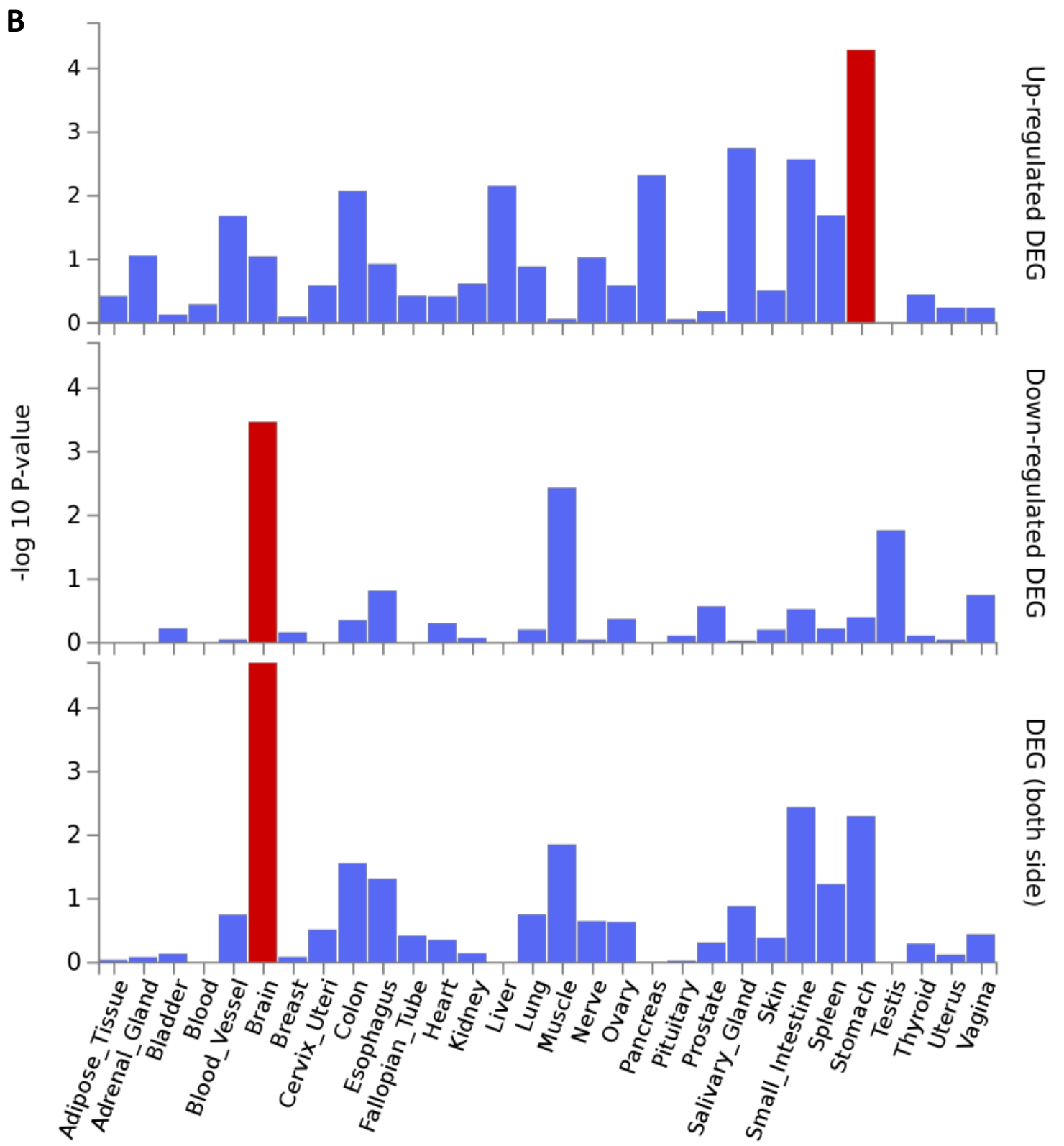

Fig. 7. FUMA GENE2FUNC analysis of 354 glycosylation enzymes and related genes in humans revealed a specific downregulation in the brain. A) Heat map demonstrating expression pattern of all glycosylation genes in humans, with a black bar above the 13 columns representing brain regions. Full-size file available in supplement. B) Tissue specific analysis showing down-regulation in the brain compared to all other 29 tissue types, with significantly enriched DEG sets $\left(P_{\text {bon }}<0.05\right)$ highlighted in red. 